\title{
SOME CHARACTERIZATIONS OF MIXED RENEWAL PROCESSES
}

\author{
Demetrios P. Lyberopoulos*, ${ }^{*}$ - Nikolaos D. Macheras** \\ (Communicated by Gejza Wimmer)
}

\begin{abstract}
Some characterizations of mixed renewal processes in terms of exchangeability and of different types of regular conditional probabilities are given. As a consequence, an existence result for mixed renewal processes, providing also a new construction for them, is obtained. As an application, some concrete examples of constructing such processes are presented and the corresponding regular conditional probabilities are explicitly computed.
\end{abstract}

$$
\text { (C) } 2022
$$

Mathematical Institute Slovak Academy of Sciences

\section{Introduction}

Although mixed renewal processes (MRPs for short) have not been studied in the literature as extensively as their mixed Poisson counterparts, they certainly possess their own merit, which has become more important during the last decades; we refer, for instance, to the pioneering paper of Huang [11] as well as to the earlier work of Segerdahl 22] with a more applied flavor. The latter two contributions are indicative for the two main reasons that explain the importance of MRPs: they may serve, on the one hand, as a useful tool for modeling real life situations, such as those emerging in actuarial practice (cf. e.g., 22: p. 164-165], where experience rating schemes on motor vehicle insurance are discussed) and on the other as a source of challenging theoretical problems, since they are generalizations of mixed Poisson processes (MPPs for short) and closely connected with the concept of exchangeable stochastic processes (cf. e.g., [1]).

Likewise, the motivation of the current work is two-fold: the main one refers to the introduction and investigation of a definition of MRPs which is both less technical than the definitions of MRPs usually met in the literature (see e.g., Grandell [8] and Huang [11]) and in line with their counterparts referring to mixed Poisson processes (MPPs for short), especially with that of MPPs with parameter $\Theta$ (cf. e.g., 21: Chapter 4] for the definition). Such a definition seems to be a

2020 Mathematics Subject Classification: Primary 60G55; Secondary 60G09, 28A50, 60A10, 60G05, 60K05, 91B30.

Keywords: mixed renewal process, regular conditional probability, product regular conditional probability, subfield regular conditional probability, exchangeability.

D.P.L. would like to aknowledge that the main part of this work was conducted at the Department of Statistics and Insurance Science in the University of Piraeus. The author is also indebted to the Public Benefit Foundation Alexander S. Onassis, which supported this research, under the Programme of Scholarships for Hellenes. D.P.L. would also like to state that this work should not be reported as representing the views of the Hellenic Statistical Authority (ELSTAT). The views expressed are those of the authors and do not necessarily reflect those of ELSTAT.

c Corresponding author. 
proper/more natural one as it involves explicitly the structural parameter $\Theta$, which is usually essential in the study of risk-theoretical problems.

In fact, this definition was exploited in [16] and 17], where the equivalence of various existing definitions of MPPs is proved and the problem of examining whether within the larger class of extended MRPs (see [17. Definition 3.2] for the definition), every Markov process is a mixed Poisson one with a random variable as mixing parameter is addressed, respectively. Moreover, the main outcomes of this work may find applications to problems related to life times of components (see also 11: p. 17-18]) and especially to the interplay between financial and actuarial pricing of insurance, see, e.g., 24 and compare it with the recent work of Lyberopoulos \& Macheras [15, where the problem of arbitrage-free pricing of an inhomogeneous portfolio of insurance risks was made possible, generalizing the financial pricing of insurance approach, introduced by Delbean \& Haezendonck $[2]$ in the framework of classical risk theory.

Since conditioning is involved in the definition of MRPs introduced in this paper, it is reasonable to ask about the structural role of regular conditional probabilities (rcps for short) in this field. For this reason, in Section 3 , we recall the definitions of different types of rcps and provide a characterization of MRPs via rcps (see Proposition 3.1), which extends a basic result, i.e., Proposition 4.4 of our previous work 13. By means of this result, we can reduce MRPs under a probability measure $P$ with parameter $\Theta$ to ordinary renewal processes under the corresponding regular conditional probabilities of $P$ over $P_{\Theta}$ consistent with $\Theta$, showing in this way that Definition 3.1 is the natural one for MRPs.

The second definition of MRPs investigated in this paper is due to Huang [11, see Definition 4.1. In Section 4, we first obtain some characterizations of exchangeability in terms of different types of rcps, see Theorem 4.2 and Corollary 4.2.1. As a consequence, in Theorem 4.3 some further characterizations of MRPs in terms of exchangeability and rcps are deduced. Theorem 4.3 provides amongst others a detailed discussion of the relation between the two definitions of MRPs and shows that in most cases appearing in applications both definitions coincide.

In Section 5 , we first give some examples to show that some of the assumptions of Theorems 4.2 and 4.3 are essential for the validity of all the equivalences obtained therein (see Examples 1 to 2. Next, we provide a construction of canonical probability spaces admitting MRPs (see Example 3), extending a similar construction for MPPs, see 14: Theorem 3.1]. As an application, we give concrete examples of MRPs satisfying all assumptions of Theorem 4.3 and we compute the corresponding rcps explicitly.

\section{Preliminaries}

By $\mathbb{N}$ is denoted the set of all natural numbers and $\mathbb{N}_{0}:=\mathbb{N} \cup\{0\}$. The symbol $\mathbb{R}$ stands for the set of all real numbers, while $\mathbb{R}_{+}:=\{x \in \mathbb{R}: x \geq 0\}$. If $d \in \mathbb{N}$, then $\mathbb{R}^{d}$ denotes the Euclidean space of dimension $d$.

Given a probability space $(\Omega, \Sigma, P)$, a set $N \in \Sigma$ with $P(N)=0$ is called a $P$-null set (or a null set for simplicity). The family of all $P$-null sets is denoted by $\Sigma_{0}$. For any two $\Sigma$-T-measurable maps $X, Y: \Omega \rightarrow \Upsilon$, we write $X=Y P$-a.s., if $\{X \neq Y\} \in \Sigma_{0}$. For the definitions of real-valued random variables, random variables and random vectors, we refer to Cohn [1. pp. 308 and 318].

If $A \subseteq \Omega$, then $A^{c}:=\Omega \backslash A$, while $\chi_{A}$ denotes the indicator (or characteristic) function of the set $A$. For a map $f: \Omega \rightarrow \Upsilon$ and for a non-empty set $A \subseteq \Omega$ denote by $f\lceil A$ the restriction of $f$ to $A$. The identity map from $\Omega$ onto itself is denoted by id $\Omega$. The $\sigma$-algebra generated by a family $\mathcal{G}$ of subsets of $\Omega$ is denoted by $\sigma(\mathcal{G})$. 
For any Hausdorff topology $\mathfrak{T}$ on $\Omega$ by $\mathfrak{B}(\Omega)$ is denoted the Borel $\sigma$-algebra on $\Omega$, i.e., the $\sigma$-algebra generated by $\mathfrak{T}$. By $\mathfrak{B}:=\mathfrak{B}(\mathbb{R}), \mathfrak{B}_{d}:=\mathfrak{B}\left(\mathbb{R}^{d}\right)$ and $\mathfrak{B}_{\mathbb{N}}:=\mathfrak{B}\left(\mathbb{R}^{\mathbb{N}}\right)$ is denoted the Borel $\sigma$-algebra of subsets of $\mathbb{R}, \mathbb{R}^{d}$ and $\mathbb{R}^{\mathbb{N}}$, respectively, while $\mathcal{L}^{1}(P)$ stands for the family of all realvalued $P$-integrable functions on $\Omega$. Functions that are $P$-a.s. equal are not identified. We write $\mathbb{E}_{P}[X \mid \mathcal{F}]$ for a version of a conditional expectation (under $P$ ) of $X \in \mathcal{L}^{1}(P)$ given a $\sigma$-subalgebra $\mathcal{F}$ of $\Sigma$, while for $X:=\chi_{E} \in \mathcal{L}^{1}(P)$ with $E \in \Sigma$, we set $P(E \mid \mathcal{F}):=\mathbb{E}_{P}\left[\chi_{E} \mid \mathcal{F}\right]$.

Given two probability spaces $(\Omega, \Sigma, P)$ and $(\Upsilon, T, Q)$, we denote by $\sigma\left(\left\{X_{i}\right\}_{i \in I}\right)$ the $\sigma$-algebra generated by a family $\left\{X_{i}\right\}_{i \in I}$ of $\Sigma$-T-measurable maps from $\Omega$ into $\Upsilon$, while if $X$ is a $\Sigma$-T-measurable map then $P_{X}$ stands for the image measure of $P$ under $X$. If $X$ is a $d$-dimensional random vector on $\Omega$, we write $P_{X}=\mathbf{K}(\theta)$ meaning that $X$ is distributed according to the law $\mathbf{K}(\theta)$, where $\theta \in \mathbb{R}^{d}$. In particular, $\mathbf{P}(\theta), \mathbf{E x p}(\theta)$ and $\mathbf{G a}(\gamma, \alpha)$, where $\theta, \gamma, \alpha$ are positive parameters, stand for the law of Poisson, exponential and gamma distribution, respectively (cf, e.g., [21]).

By $(\Omega \times \Upsilon, \Sigma \otimes T, P \otimes Q)$ is denoted the product probability space of $(\Omega, \Sigma, P)$ and $(\Upsilon, T, Q)$, and by $\pi_{\Omega}$ and $\pi_{\Upsilon}$ the canonical projections from $\Omega \times \Upsilon$ onto $\Omega$ and $\Upsilon$, respectively.

Given two measurable spaces $(\Omega, \Sigma)$ and $(\Upsilon, T)$, a $\Sigma$-T-Markov kernel is a function $k$ from $T \times \Omega$ into $\mathbb{R}$ satisfying the following conditions:

(k1) The set-function $k(\cdot, \omega)$ is a probability measure on $T$ for any fixed $\omega \in \Omega$.

(k2) The function $\omega \mapsto k(B, \omega)$ is $\Sigma$-measurable for any fixed $B \in T$.

In particular, given a probability measure $Q$ on $T$, a family $\left\{P_{y}\right\}_{y \in \Upsilon}$ of probability measures on $\Sigma$ is called a regular conditional probability (rcp for short) of $P$ over $Q$ if

(d1) for each $D \in \Sigma$ the map $y \mapsto P_{y}(D)$ is $T$-measurable;

(d2) $\int P_{y}(D) Q(d y)=P(D)$ for each $D \in \Sigma$.

If $f: \Omega \rightarrow \Upsilon$ is an inverse-measure-preserving function (i.e., $P\left(f^{-1}(B)\right)=Q(B)$ for each $B \in T$ ), an rcp $\left\{P_{y}\right\}_{y \in \Upsilon}$ of $P$ over $Q$ is called consistent with $f$ if for each $B \in T$, the equality $P_{y}\left(f^{-1}(B)\right)=1$ holds for $Q$-almost every $y \in B$.

We could use the term of disintegration instead, but it seems that it is better to reserve that term to the general case when $P_{y}$ 's may be defined on different domains (see [19]).

Recall that if $\Sigma$ is countably generated and $P$ is perfect (see e.g., [1. p. 102] and [5 p. 291], respectively, for the definitions), then there always exists an $\operatorname{rcp}\left\{P_{y}\right\}_{y \in \Upsilon}$ of $P$ over $Q$ consistent with any inverse-measure-preserving map $f$ from $\Omega$ into $\Upsilon$ providing that $T$ is countably generated (see 5: Theorems 6 and 3]) and a subfield rcp (see [5. Theorems 6 and 2] as well as [5: Section 2] for the definition). So, in most cases appearing in applications these two types of rcps always exist. A Polish space, that is a topological space homeomorphic to a complete separable metric space, is such a case.

Let now $(\Psi, Z)$ be a measurable space and let $X: \Omega \rightarrow \Upsilon$ and $\Theta: \Omega \rightarrow \Psi$ be a $\Sigma$-T-measurable and a $\Sigma$ - $Z$-measurable map, respectively. A conditional distribution of $X$ over $\Theta$, denoted by $P_{X \mid \Theta}$, is a $\sigma(\Theta)$-T-Markov kernel $k$ satisfying for each $B \in T$ condition

$$
k(B, \cdot)=P\left(X^{-1}(B) \mid \Theta\right)(\cdot) \quad P\lceil\sigma(\Theta) \text {-a.s.. }
$$

Note that if $\Upsilon$ is a Polish space then a conditional distribution of $X$ over $\Theta$ always exists (cf. e.g., [4. Theorem 10.2.2]).

Clearly, for every $Z$-T-Markov kernel $k$, the map $K(\Theta)$ from $T \times \Omega$ into $\mathbb{R}$ defined by means of

$$
K(\Theta)(B, \omega):=(k(B, \cdot) \circ \Theta)(\omega) \quad \text { for any } B \in T \text { and } \omega \in \Omega
$$


is a $\sigma(\Theta)$-T-Markov kernel. In particular, for $(\Upsilon, T)=(\mathbb{R}, \mathfrak{B})$ its associated probability measures $k(\cdot, \theta)$ for $\theta=\Theta(\omega)$ with $\omega \in \Omega$ are distributions on $\mathfrak{B}$ and so, we may write $\mathbf{K}(\theta)(\cdot)$ instead of $k(\cdot, \theta)$. Consequently, in this case $K(\Theta)$ will be denoted by $\mathbf{K}(\Theta)$.

For any $\sigma$-subalgebra $\mathcal{F}$ of $\Sigma$, we say that two $\mathcal{F}$-T-Markov kernels $k_{i}$, for $i \in\{1,2\}$, are $P \mid \mathcal{F}$-equivalent and, we write $k_{1}=k_{2} P \mid \mathcal{F}$-a.s., if there exists a $P$-null set $N \in \mathcal{F}$ such that for any $\omega \notin N$ and $B \in T$ the equality $k_{1}(B, \omega)=k_{2}(B, \omega)$ holds true.

From now on $(\Omega, \Sigma, P)$ is a probability space, while $(\Upsilon, T)$ and $(\Psi, Z)$ are measurable spaces, all of them arbitrary but fixed.

\section{Characterizations of mixed renewal processes via regular conditional probabilities}

Let $\left\{N_{t}\right\}_{t \in \mathbb{R}_{+}}$be a $(P-)$ counting process with exceptional $(P-)$ null set $\Omega_{N}$ (cf. e.g., 21: p. 17] for the definition). Without loss of generality, we may and do assume that $\Omega_{N}=\emptyset$. Denote by $\left\{T_{n}\right\}_{n \in \mathbb{N}_{0}}$ and $\left\{W_{n}\right\}_{n \in \mathbb{N}}$ the arrival process and interarrival process, respectively, associated with $\left\{N_{t}\right\}_{t \in \mathbb{R}_{+}}$(cf. e.g., 21 ; p. 6] for the definitions).

Recall now that for a $\Sigma$-Z-measurable map $\Theta$ from $\Omega$ onto $\Psi$, a family $\left\{X_{i}\right\}_{i \in I}$ of $\Sigma$-T-measurable maps from $\Omega$ into $\Upsilon$ is $P$-conditionally (stochastically) independent given $\Theta$ if

$$
P\left(\bigcap_{j=1}^{n} X_{i_{j}}^{-1}\left(B_{j}\right) \mid \Theta\right)=\prod_{j=1}^{n} P\left(X_{i_{j}}^{-1}\left(B_{j}\right) \mid \Theta\right) \quad P\lceil\sigma(\Theta) \text {-a.s. }
$$

whenever $i_{1}, \ldots, i_{n}$ are distinct members of $I$ and $B_{j} \in T$ for every $j \leq n$. The family $\left\{X_{i}\right\}_{i \in I}$ is $P$-conditionally identically distributed given $\Theta$, if

$$
P\left(F \cap X_{i}^{-1}(B)\right)=P\left(F \cap X_{j}^{-1}(B)\right)
$$

whenever $i, j \in I, F \in \sigma(\Theta)$ and $B \in T$.

Throughout what follows, unless it is stated otherwise, $\Theta$ is a $\Sigma$-Z-measurable map from $\Omega$ into $\Psi$, and we simply write "conditionally" in the place of "conditionally given $\Theta$ " whenever conditioning refers to $\Theta$. Moreover, $\left\{N_{t}\right\}_{t \in \mathbb{R}_{+}}$is a counting process and without loss of generality, we may and do assume that $\Omega_{N}=\emptyset$.

The counting process $\left\{N_{t}\right\}_{t \in \mathbb{R}_{+}}$is said to be a $P$-renewal process with interarrival time distribution $\mathbf{K}\left(\theta_{0}\right)$, where $\theta_{0} \in \mathbb{R}^{d}$ is a parameter (or a $\left(P, \mathbf{K}\left(\theta_{0}\right)\right.$ )-RP for short), if its associated interarrival times $W_{n}, n \in \mathbb{N}$, are independent and $\mathbf{K}\left(\theta_{0}\right)$-distributed under the probability measure $P$.

Definition 3.1. The counting process $\left\{N_{t}\right\}_{t \in \mathbb{R}_{+}}$is said to be a mixed renewal process on $(\Omega, \Sigma, P)$ with parameter the $d$-dimensional $(d \in \mathbb{N})$ random vector $\Theta$ and interarrival time conditional distribution $\mathbf{K}(\Theta)$ (or a $(P, \mathbf{K}(\Theta))$-MRP for short), if $\left\{W_{n}\right\}_{n \in \mathbb{N}}$ is $P$-conditionally independent and

for all $n \in \mathbb{N}$.

$$
P_{W_{n} \mid \Theta}=\mathbf{K}(\Theta) \quad P\lceil\sigma(\Theta) \text {-a.s. }
$$

In particular, for $(\Psi, Z)=(\mathbb{R}, \mathfrak{B})$ and $P_{\Theta}((0, \infty))=1$ a counting process $\left\{N_{t}\right\}_{t \in \mathbb{R}_{+}}$is a $P$-mixed Poisson process on $(\Omega, \Sigma, P)$ (or a $P$-MPP for short) with parameter the random variable $\Theta$, if it has $P$-conditionally stationary independent increments (cf. e.g., [21: Section 4.1, p. 86] for the definition), such that

$$
P_{N_{t} \mid \Theta}=\mathbf{P}(t \Theta) \quad P\lceil\sigma(\Theta) \text {-a.s. }
$$

holds true for each $t \in(0, \infty)$. 
Note that, for $d=1, P_{\Theta}((0, \infty))=1$ and $\mathbf{K}(\Theta)=\mathbf{E x p}(\Theta) P\lceil\sigma(\Theta)$-a.s. the $(P, \mathbf{K}(\Theta))$-MRP $\left\{N_{t}\right\}_{t \in \mathbb{R}_{+}}$becomes a $P$-MPP with parameter $\Theta$ (see 13. Proposition 4.5]).

Remarks 1. (a) Let $\left\{P_{y}\right\}_{y \in \Upsilon}$ be an $\operatorname{rcp}$ of $P$ over $Q$, and let $f$ be an inverse-measure-preserving map from $\Omega$ into $\Upsilon$. Then the following are equivalent:

$$
\begin{aligned}
& \left\{P_{y}\right\}_{y \in \Upsilon} \text { is consistent with } f . \\
& P\left(A \cap f^{-1}(B)\right)=\int_{B} P_{y}(A) Q(\mathrm{~d} y) \text { for each } A \in \Sigma \text { and } B \in T .
\end{aligned}
$$

For each $A \in \Sigma \quad \mathbb{E}_{P}\left[\chi_{A} \mid \sigma(f)\right]=P \bullet(A) \circ f \quad P\lceil\sigma(f)$-a.s..

(b) Let $X$ be a $\Sigma$-T-measurable map from $\Omega$ into $\Upsilon$, let $\left\{P_{\theta}\right\}_{\theta \in \Psi}$ be an rcp of $P$ over $P_{\Theta}$ consistent with $\Theta$, and let $k$ be a $Z$-T-Markov kernel. If $k(\cdot, \theta)$ is the distribution of $X$ under $P_{\theta}$ for $\theta \in \Psi$, then the map $K(\Theta)$ is a conditional distribution of $X$ given $\Theta$, since by condition (3.3) of (a), we get for $A=X^{-1}(B)$ with $B \in T$ that $P_{X \mid \Theta}(B, \cdot)=K(\Theta)(B, \cdot) P\lceil\sigma(\Theta)$-a.s..

(c) Conversely, in the special case where $\Sigma$ is countably generated and $(\Upsilon, T)=(\mathbb{R}, \mathfrak{B})$, given $\left\{P_{\theta}\right\}_{\theta \in \Psi}$ as in (b), we get that for each conditional distribution $\mathbf{K}(\Theta)$ of $X$ given $\Theta$, there exists an essentially unique probability distribution $\left(P_{\theta}\right)_{X}$ of $X$, for $\theta \in \Psi$, such that for each $B \in \mathfrak{B}$, we have

$$
\mathbf{K}(\Theta)(B, \cdot)=\left(P_{\bullet}\right)_{X}(B) \circ \Theta \quad P \uparrow \sigma(\Theta) \text {-a.s.. }
$$

In fact, by applying a monotone class argument, it can be easily seen that the rcp is essentially unique in the sense that if $\left\{P_{\theta}^{\prime}\right\}_{\theta \in \Psi}$ is any other rcp of $P$ over $P_{\Theta}$ which is consistent with $\Theta$, then $P_{\theta}=P_{\theta}^{\prime}$ for $P_{\Theta}$-almost all $\left(P_{\Theta}\right.$-a.a. for short) $\theta \in \Psi$. But the consistency of $\left\{P_{\theta}\right\}_{\theta \in \Psi}$ together with (a) yields that condition 3.3 holds true; hence setting $A=X^{-1}(B)$ with $B \in \mathfrak{B}$, we deduce that $\mathbf{K}(\Theta)(B, \cdot)=\left(P_{\bullet}\right)_{X}(B) \circ \Theta P\lceil\sigma(\Theta)$-a.s..

If no confusion arises, we denote $\left(P_{\theta}\right)_{X}$ by $\mathbf{K}(\theta)$ for $\theta \in \Psi$.

Throughout what follows, the conditional distribution $\mathbf{K}(\Theta)$ involving in Remark 1(c) will be considered together with the distributions $\mathbf{K}(\theta)$, for $\theta \in \Psi$, associated with $\mathbf{K}(\Theta)$ as in the above remark, without any additional comments.

For the remainder of this section, $\left\{P_{\theta}\right\}_{\theta \in \Psi}$ is an rcp of $P$ over $P_{\Theta}$ consistent with $\Theta$ and $\left\{X_{i}\right\}_{i \in I}$ is a non empty family of $\Sigma$-T-measurable maps from $\Omega$ into $\Upsilon$.

The next result extends Lemma 4.3 from 13 .

LemMA 3.1. If $\left\{k_{i}\right\}_{i \in I}$ is a non empty family of Z-T-Markov kernels, then for each $i \in I$ and for any fixed $B \in T$ the following conditions are equivalent:

(i) $P_{X_{i} \mid \Theta}(B, \cdot)=K_{i}(\Theta)(B, \cdot) \quad P \uparrow \sigma(\Theta)$-a.s.;

(ii) $P_{\theta}\left(X_{i}^{-1}(B)\right)=k_{i}(B, \theta)$ for $P_{\Theta}$-a.a. $\theta \in \Psi$.

In particular, the same remains true if $K_{i}(\Theta)(B, \cdot)$ and $k_{i}(B, \theta)$ are independent of $i$ for all $B \in T$ and $P_{\Theta}$-a.a. $\theta \in \Psi$. 
Pr o of. Let us fix on arbitrary $i \in I$. For all $B \in T$ and $D \in Z$, we obtain that

$$
\begin{array}{cc}
\int_{\Theta^{-1}(D)} P_{X_{i} \mid \Theta}(B, \cdot) \mathrm{d} P=\int_{\Theta^{-1}(D)} K_{i}(\Theta)(B, \cdot) \mathrm{d} P \\
\Longleftrightarrow \quad \int_{\Theta^{-1}(D)} \mathbb{E}_{P}\left[\chi_{X_{i}^{-1}(B)} \mid \sigma(\Theta)\right] \mathrm{d} P=\int_{\Theta^{-1}(D)} k_{i}(B, \cdot) \circ \Theta \mathrm{d} P \\
\stackrel{\int_{D}}{\Longleftrightarrow} P_{\theta}\left(X_{i}^{-1}(B)\right) P_{\Theta}(\mathrm{d} \theta)=\int_{D} k_{i}(B, \theta) P_{\Theta}(\mathrm{d} \theta) .
\end{array}
$$

Consequently, the equivalence of assertions (i) and (ii) follows.

Lemma 3.2. Let $\left\{k_{i}\right\}_{i \in I}$ be as in Lemma 3.1. Suppose that $I$ is countable and $T$ is countably generated. Then the following are equivalent:

(i) Condition $P_{X_{i} \mid \Theta}=K_{i}(\Theta) P\lceil\sigma(\Theta)$-a.s. holds true for each $i \in I$;

(ii) for $P_{\Theta}$-a.a. $\theta \in \Psi$ condition $P_{\theta} \circ X_{i}^{-1}=k_{i}(\cdot, \theta)$ holds true for each $i \in I$.

In particular, the same remains true if $K_{i}(\Theta)$ and $k_{i}(\cdot, \theta)$ are independent of $i$.

Proof. If (i) holds true, we then get by Lemma 3.1 that for each $i \in I$ and $B \in T$ condition

$$
P_{\theta}\left(X_{i}^{-1}(B)\right)=k_{i}(B, \theta) \quad \text { for } P_{\Theta} \text {-a.a. } \theta \in \Psi
$$

is satisfied, which is equivalent to the fact that

$$
\forall i \in I \quad \forall B \in T \exists \widetilde{L}_{I, i, B} \in Z_{0} \forall \theta \notin \widetilde{L}_{I, i, B} \quad P_{\theta}\left(X_{i}^{-1}(B)\right)=k_{i}(B, \theta),
$$

where $Z_{0}:=\left\{L \in Z: P_{\Theta}(L)=0\right\}$.

Since $I$ is countable and $T$ is countably generated, letting

$$
\widetilde{L}_{I}:=\bigcup_{B \in \mathcal{G}_{T}} \bigcup_{i \in I} \widetilde{L}_{I, i, B}
$$

where $\mathcal{G}_{T}$ is a countable generator of $T$, and applying a monotone class argument, we find a $P_{\Theta}$-null set $\widetilde{L}_{I} \in Z$ such that for any $\theta \notin \widetilde{L}_{I}$ the equality $P_{\theta}\left(X_{i}^{-1}(B)\right)=k_{i}(B, \theta)$ holds true. So assertion (ii) follows.

Applying a similar reasoning, we obtain the converse implication.

The following result extends Lemma 4.1 from 13 .

Lemma 3.3. Let $I$ be countable and $T$ countably generated. Then the family $\left\{X_{i}\right\}_{i \in I}$ is $P$-conditionally independent if and only if for $P_{\Theta}$-a.a. $\theta \in \Psi$ it is $P_{\theta}$-independent.

Pr o of. Assume that $\left\{X_{i}\right\}_{i \in I}$ is $P$-conditionally independent. Then according to Remark 11(a) and following the same reasoning as in the proof of [13. Lemma 4.1], we get that

$$
\int_{D} P_{\theta}\left(\bigcap_{j=1}^{m}\left\{X_{i_{j}} \in B_{j}\right\}\right) P_{\Theta}(\mathrm{d} \theta)=\int_{D} \prod_{j=1}^{m} P_{\theta}\left(\left\{X_{i_{j}} \in B_{j}\right\}\right) P_{\Theta}(\mathrm{d} \theta)
$$

whenever $D \in Z, m \in \mathbb{N}, i_{1}, \ldots, i_{m} \in I$ are distinct, and $B_{1}, \ldots, B_{m} \in T$, equivalently that for each $m \in \mathbb{N}$, for all $i_{1}, \ldots, i_{m} \in I$ distinct and for all $B_{1}, \ldots, B_{m} \in T$ there exists a $P_{\Theta}$-null set $L_{I, m, i_{1}, \ldots, i_{m}, B_{1}, \ldots, B_{m}} \in Z$ such that for every $\theta \notin L_{I, m, i_{1}, \ldots, i_{m}, B_{1}, \ldots, B_{m}}$ condition

$$
P_{\theta}\left(\bigcap_{j=1}^{m}\left\{X_{i_{j}} \in B_{j}\right\}\right)=\prod_{j=1}^{m} P_{\theta}\left(\left\{X_{i_{j}} \in B_{j}\right\}\right)
$$


holds true. Without loss of generality, we may and do assume that $m=2$. Since $T$ is countably generated, applying successively two monotone class arguments, we get that there exists a $P_{\Theta}$-null set $L_{I} \in Z$ such that for any $\theta \notin L_{I}$ condition (3.4) holds true for $m=2$, for each $i_{1}, i_{2} \in I$ with $i_{1} \neq i_{2}$ and for each $B_{1}, B_{2} \in T$; hence $\left\{X_{i}\right\}_{i \in I}$ is $P_{\theta}$-independent for any $\theta \notin L_{I}$. Since the inverse implication is clear, this completes the proof.

Lemma 3.4. Let $I$ be countable and $T$ countably generated. Then the following hold true:

(i) The family $\left\{X_{i}\right\}_{i \in I}$ is $P$-conditionally identically distributed if and only if for $P_{\Theta}$-a.a. $\theta \in \Psi$ it is $P_{\theta}$-identically distributed.

(ii) The family $\left\{X_{i}\right\}_{i \in I}$ is $P$-conditionally i.i.d. if and only if for $P_{\Theta}$-a.a. $\theta \in \Psi$ it is $P_{\theta}$-i.i.d..

Proof. Ad (i): If $\left\{X_{i}\right\}_{i \in I}$ is $P$-conditionally identically distributed then for any two $i, j \in I$ and for each $B \in T$ the equality $P_{X_{i} \mid \Theta}(B)=P_{X_{j} \mid \Theta}(B)$ holds true $P\lceil\sigma(\Theta)$-a.s..

Then applying successively Remark 1(a) and a monotone class argument as that in the proof of Lemma 3.2 we find a $P_{\Theta}$-null set $\widetilde{L}_{I}^{\prime} \in Z$ such that for any $\theta \notin \widetilde{L}_{I}^{\prime}$ the family $\left\{X_{i}\right\}_{i \in I}$ is $P_{\theta}$-identically distributed. The inverse implication is immediate by Remark 1)(a).

Ad (ii): Assume that $\left\{X_{i}\right\}_{i \in I}$ is $P$-conditionally i.i.d.. It then follows by assertion (i) and Lemma 3.3 that there exist two $P_{\Theta}$-null sets $\widetilde{L}_{I}^{\prime}$ and $L_{I}$ in $Z$ such that for any $\theta \notin \widehat{L}_{I}:=\widetilde{L}_{I}^{\prime} \cup L_{I}$ the family $\left\{X_{i}\right\}_{i \in I}$ is $P_{\theta}$-i.i.d.. Since the inverse implication is clear, this completes the proof.

Proposition 3.1. The counting process $\left\{N_{t}\right\}_{t \in \mathbb{R}_{+}}$is a $(P, \mathbf{K}(\Theta))$-MRP if and only if for $P_{\Theta}$-a.a. $\theta \in \mathbb{R}^{d}$ it is a $\left(P_{\theta}, \mathbf{K}(\theta)\right)-R P$.

Proof. Assume that $\left\{N_{t}\right\}_{t \in \mathbb{R}_{+}}$is a $(P, \mathbf{K}(\Theta))$-MRP, i.e. that the process $\left\{W_{n}\right\}_{n \in \mathbb{N}}$ is $P$ - conditionally independent and that for all interarrival times $W_{n}$ condition $P_{W_{n} \mid \Theta}=\mathbf{K}(\Theta)$ holds true $P\left\lceil\sigma(\Theta)\right.$-a.s.. Applying now Lemmas 3.3 and 3.2 we equivalently get that there exist two $P_{\Theta}$-null sets $H_{\mathbb{N}}$ and $\widetilde{H}_{\mathbb{N}}$ in $Z$ such that for any $\theta \notin H_{*}:=H_{\mathbb{N}} \cup \widetilde{H}_{\mathbb{N}}$ the sequence $\left\{W_{n}\right\}_{n \in \mathbb{N}}$ is $P_{\theta}$-independent and $\left(P_{\theta}\right)_{W_{n}}=\mathbf{K}(\theta)$ for each $n \in \mathbb{N}$, respectively, i.e. such that $\left\{N_{t}\right\}_{t \in \mathbb{R}_{+}}$is a $\left(P_{\theta}, \mathbf{K}(\theta)\right)$-RP for any $\theta \notin H_{*}$.

Next, we need a preparatory result extending Lemmas 3.3 and 3.4 for uncountable index set. To this aim, we recall some notions more.

A filtration $\left\{\mathcal{Z}_{t}\right\}_{t \in \mathbb{R}_{+}}$is said to be right-continuous if $\mathcal{Z}_{t}=\bigcap_{s>t} \mathcal{Z}_{s}$ for any $t \in \mathbb{R}_{+}$. Let $I$ be an arbitrary subset of $\mathbb{R}_{+}$and let $\Upsilon$ be a metric space. We say that the family $\left\{X_{i}\right\}_{i \in I}$ of $\Sigma$ - $\mathfrak{B}(\Upsilon)$ measurable maps from $\Omega$ into $\Upsilon$ is separable, if there exists a countable set $G \subseteq I$ such that for each $\omega \in \Omega$ the set $\left\{\left(u, X_{u}(\omega)\right): u \in G\right\}$ is dense in $\left\{\left(i, X_{i}(\omega)\right): i \in I\right\}$. Any such set $G$ is called a separator (or separating set) for $\left\{X_{i}\right\}_{i \in I}$.

Remarks 2. Let $I \subseteq \mathbb{R}_{+}$and let $Q$ be a probability measure on $\Sigma$. Then the following can be easily proven:

(a) If $\left\{U_{t}\right\}_{t \in I}$ is a family of $\Sigma$ - $\mathfrak{B}(\Upsilon)$-measurable maps from $\Omega$ into $\Upsilon$, and $\left\{\mathcal{Z}_{t}\right\}_{t \in I}$ is its canonical filtration, then $\left\{U_{t}\right\}_{t \in I}$ is $Q$-independent if and only if for every bounded $\mathfrak{B}(\Upsilon)$-measurable realvalued function $f$ on $\Upsilon$ the equality

$$
\mathbb{E}_{Q}\left[\chi_{A} f\left(U_{t}\right)\right]=Q(A) \mathbb{E}_{Q}\left[f\left(U_{t}\right)\right]
$$

holds true for each $s, t \in I$ with $s<t$ and for each $A \in \mathcal{Z}_{s}$.

(b) If $U_{1}$ and $U_{2}$ are two $\Sigma$ - $\mathfrak{B}(\Upsilon)$-measurable maps from $\Omega$ into $\Upsilon$, then they are $Q$-identically distributed if and only if $\mathbb{E}_{Q}\left[f\left(U_{1}\right)\right]=\mathbb{E}_{Q}\left[f\left(U_{2}\right)\right]$ for every bounded $\mathfrak{B}(\Upsilon)$-measurable real-valued function $f$ on $\Upsilon$. 
Proposition 3.2. Let $\Upsilon$ be a Polish space, let $\left\{X_{t}\right\}_{t \in \mathbb{R}_{+}}$be a family of $\Sigma-\mathfrak{B}(\Upsilon)$-measurable maps from $\Omega$ into $\Upsilon$ and let $\left\{\mathcal{H}_{t}\right\}_{t \in \mathbb{R}_{+}}$be its canonical filtration. If the family $\left\{X_{t}\right\}_{t \in \mathbb{R}_{+}}$is separable with separator $\mathbb{Q}_{+}$, then the following hold true:

(i) If $\left\{\mathcal{H}_{t}\right\}_{t \in \mathbb{R}_{+}}$is right-continuous, then $\left\{X_{t}\right\}_{t \in \mathbb{R}_{+}}$is P-conditionally independent if and only if for $P_{\Theta}$-a.a. $\theta \in \Psi$ it is $P_{\theta}$-independent.

(ii) The family $\left\{X_{t}\right\}_{t \in \mathbb{R}_{+}}$is $P$-conditionally identically distributed if and only if for $P_{\Theta}$-a.a. $\theta \in \Psi$ it is $P_{\theta}$-identically distributed.

(iii) If $\left\{\mathcal{H}_{t}\right\}_{t \in \mathbb{R}_{+}}$is right-continuous, then $\left\{X_{t}\right\}_{t \in \mathbb{R}_{+}}$is P-conditionally i.i.d. if and only if for $P_{\Theta}$-a.a. $\theta \in \Psi$ it is $P_{\theta}$-i.i.d..

Pro of. Ad (i): The "if" implication follows as in the proof of Lemma 4.1 from 13. For the "only if" part, note that our assumptions for $\left\{X_{t}\right\}_{t \in \mathbb{R}_{+}}$and $\left\{\mathcal{H}_{t}\right\}_{t \in \mathbb{R}_{+}}$imply $\mathcal{H}_{s}=\sigma\left(\left\{X_{u}\right\}_{u \in \mathbb{Q}_{+}, u \leq s}\right)=$ $\bigcap \mathcal{H}_{s^{\prime}}$ for each $s \in \mathbb{R}_{+}$. $s^{\prime} \in \mathbb{Q}_{+}, s^{\prime}>s$

(a) It follows by Lemma 3.3 that there exists a $P_{\Theta}$-null set $O_{\mathbb{Q}_{+}} \in Z$ such that for any $\theta \notin O_{\mathbb{Q}_{+}}$ condition (3.4) holds true with $\mathbb{Q}_{+}$and $\mathfrak{B}(\Upsilon)$ in the place of $I$ and $T$, respectively.

Throughout this proof fix on an arbitrary $\theta \notin O_{\mathbb{Q}_{+}}$. Then condition (3.4) together with Remark 2(a) implies that for all $s, t \in \mathbb{Q}_{+}$with $s<t$, for every bounded $\mathfrak{B}(\Upsilon)$-measurable real-valued function $f$ on $\Upsilon$ and for each $A \in \mathcal{H}_{s}$, we have

$$
\mathbb{E}_{P_{\theta}}\left[\chi_{A} f\left(X_{t}\right)\right]=P_{\theta}(A) \mathbb{E}_{P_{\theta}}\left[f\left(X_{t}\right)\right] .
$$

If we take $s, t \in \mathbb{R}_{+}$with $s<t$ and write $(3.6)$ for $s^{\prime}, t^{\prime} \in \mathbb{Q}_{+}$with $s^{\prime}<t^{\prime}$ and then let $s^{\prime} \downarrow s$ and $t^{\prime} \downarrow t$, the separability of $\left\{X_{t}\right\}_{t \in \mathbb{R}_{+}}$together with an application of Lebesgue's Dominated Convergence Theorem yields that for all $A \in \bigcap_{s^{\prime} \in \mathbb{Q}_{+}, s^{\prime}>s} \mathcal{H}_{s^{\prime}}=\mathcal{H}_{s}$ and for every bounded continuous real-valued function $f$ on $\Upsilon$ condition (3.6) holds true.

(b) Let $s, t \in \mathbb{R}_{+}$with $s<t$ and let $f$ be a function as in $(3.5)$. Then for each $n \in \mathbb{N}$ there exists a bounded continuous real-valued function $g_{n}$ on $\Upsilon$ satisfying the inequality $\int\left|g_{n}-f\right| d\left(P_{\theta}\right)_{X_{t}} \leq \frac{1}{n}$ (cf. e.g., 7: Proposition 415P]); hence there exists a sequence $\left\{g_{n}\right\}_{n \in \mathbb{N}}$ of bounded continuous realvalued functions on $\Upsilon$ such that condition $\lim _{n \rightarrow \infty} \int \chi_{A}\left(\left|g_{n}-f\right| \circ X_{t}\right) \mathrm{d} P_{\theta}=0$ holds true for all $A \in \mathcal{H}_{s}$, implying together with (a) that $\mathbb{E}_{P_{\theta}}\left[\chi_{A} f\left(X_{t}\right)\right]=\lim _{n \rightarrow \infty} \mathbb{E}_{P_{\theta}}\left[\chi_{A} g_{n}\left(X_{t}\right)\right]=\lim _{n \rightarrow \infty} P_{\theta}(A) \mathbb{E}_{P_{\theta}}\left[g_{n}\left(X_{t}\right)\right]=$ $P_{\theta}(A) \mathbb{E}_{P_{\theta}}\left[f\left(X_{t}\right)\right]$ for all $A \in \mathcal{H}_{s}$; hence by Remark $2\left(\right.$ a), we get that $\left\{X_{t}\right\}_{t \in \mathbb{R}_{+}}$is $P_{\theta}$-independent, which proves (i).

Ad (ii): The "if" implication is immediate by Remark11(b). For the "only if" part, assume that $\left\{X_{t}\right\}_{t \in \mathbb{R}_{+}}$is $P$-conditionally identically distributed.

(c) Since $\left\{X_{t}\right\}_{t \in \mathbb{R}_{+}}$is $P$-conditionally identically distributed, we get that for any two $s, t \in \mathbb{Q}_{+}$ and for each $B \in \mathfrak{B}(\Upsilon)$ the equality $P_{X_{t} \mid \Theta}(B)=P_{X_{s} \mid \Theta}(B)$ holds $P\lceil\sigma(\Theta)$-a.s.. The latter together with Lemma 3.4 (i) yields the existence of a $P_{\Theta^{-}}$null set $\widetilde{O}_{\mathbb{Q}_{+}} \in Z$ such that for any $\theta \notin \widetilde{O}_{\mathbb{Q}_{+}}$and for all $s, t \in \mathbb{Q}_{+}$condition $\left(P_{\theta}\right)_{X_{t}}=\left(P_{\theta}\right)_{X_{s}}$ holds true, which by Remark 2(b) equivalently yields that for any $\theta \notin \widetilde{O}_{\mathbb{Q}_{+}}$, for every function $f$ as in the above remark, and for all $s, t \in \mathbb{Q}_{+}$, we have $\mathbb{E}_{P_{\theta}}\left[f\left(X_{t}\right)\right]=\mathbb{E}_{P_{\theta}}\left[f\left(X_{s}\right)\right]$.

Till the end of the proof of (ii), fix on an arbitrary $\theta \notin \widetilde{O}_{\mathbb{Q}_{+}}$.

(d) If we take $s, t \in \mathbb{R}_{+}$and if we write the last equality for $s^{\prime}, t^{\prime} \in \mathbb{Q}_{+}$and then let $s^{\prime} \downarrow s$ and $t^{\prime} \downarrow t$, the separability of $\left\{X_{t}\right\}_{t \in \mathbb{R}_{+}}$together with an application of Lebesgue's Dominated Convergence Theorem yields that for every bounded continuous real-valued function $f$ on $\Upsilon$ condition $\mathbb{E}_{P_{\theta}}\left[f\left(X_{t}\right)\right]=\mathbb{E}_{P_{\theta}}\left[f\left(X_{s}\right)\right]$ holds true. 
Following now the same reasoning with that of steps (b) and (c), we obtain that the last equality is satisfied by all functions $f$ as in Remark 2 , and all $s, t \in \mathbb{R}_{+}$, which is equivalent to the fact that condition $\left(P_{\theta}\right)_{X_{t}}=\left(P_{\theta}\right)_{X_{s}}$ holds true for all $s, t \in \mathbb{R}_{+}$; hence (ii) follows.

Ad (iii): Assume that $\left\{X_{t}\right\}_{t \in \mathbb{R}_{+}}$is $P$-conditionally i.i.d. and that its canonical filtration is right-continuous. It then follows by assertions (i) and (ii) that there exist two $P_{\Theta}$-null sets $\widetilde{O}_{\mathbb{Q}_{+}}$ and $O_{\mathbb{Q}_{+}}$in $Z$ such that for any $\theta \notin \widehat{O}_{\mathbb{Q}_{+}}:=\widetilde{O}_{\mathbb{Q}_{+}} \cup O_{\mathbb{Q}_{+}}$the family $\left\{X_{t}\right\}_{t \in \mathbb{R}_{+}}$is $P_{\theta}$-i.i.d.. Since the inverse implication is clear, assertion (iii) follows.

The next result seems to be of independent interest, since it shows among others that for any counting process the property of conditionally independent increments under a probability measure can be reduced to that of independent increments under the probability measures of an rcp. The same result extends a basic one, namely [13: Proposition 4.4].

THEOREM 3.3. Let $\left\{N_{t}\right\}_{t \in \mathbb{R}_{+}}$be a counting process and let $\left\{\widetilde{\mathbf{K}}_{t}(\theta)\right\}_{t \in \mathbb{R}_{+}}$be a family of probability distributions on $\mathfrak{B}$ with parameter $\theta \in \Psi$. Then $\left\{N_{t}\right\}_{t \in \mathbb{R}_{+}}$has P-conditionally stationary independent increments such that condition

$$
P_{N_{t} \mid \Theta}=\widetilde{\mathbf{K}}_{t}(\Theta) \quad P \uparrow \sigma(\Theta) \text {-a.s. }
$$

holds true for each $t \in \mathbb{R}_{+}$if and only if for $P_{\Theta}$-a.a. $\theta \in \Psi$ it has $P_{\theta}$-stationary independent increments such that $\left(P_{\theta}\right)_{N_{t}}=\widetilde{\mathbf{K}}_{t}(\theta)$ for each $t \in \mathbb{R}_{+}$.

Pro of. Since $\left\{N_{t}\right\}_{t \in \mathbb{R}_{+}}$is a counting process it has right-continuous paths; hence it is separable with separator $\mathbb{Q}_{+}$. Note also that the canonical filtration of $\left\{N_{t}\right\}_{t \in \mathbb{R}_{+}}$is right-continuous (see 20: Theorem 25], where the proof works for any probability space not necessarily complete). Thus, all assumptions of Proposition 3.2 are fulfilled, and so, we may apply it to deduce the thesis of the theorem.

Corollary 3.3.1 (13: Proposition 4.4]). The family $\left\{N_{t}\right\}_{t \in \mathbb{R}_{+}}$is a P-MPP with parameter $\Theta$ if and only if it is a $P_{\theta}$-Poisson process with parameter $\theta$ for $P_{\Theta}$-a.a. $\theta \in \mathbb{R}$.

\section{Further characterizations}

Recall first that an infinite family $\left\{X_{i}\right\}_{i \in I}$ of $\Sigma$-T-measurable maps from $\Omega$ into $\Upsilon$ is said to be exchangeable under $P$ or $P$-exchangeable, if for each $r \in \mathbb{N}$, we have

$$
P\left(\bigcap_{k=1}^{r} X_{i_{k}}^{-1}\left(B_{k}\right)\right)=P\left(\bigcap_{k=1}^{r} X_{j_{k}}^{-1}\left(B_{k}\right)\right)
$$

whenever $i_{1}, \ldots, i_{r} \in I$ are distinct, $j_{1}, \ldots, j_{r} \in I$ are distinct, and $B_{k} \in T$ for each $k \leq r$ (cf. e.g., 77: 459C]). Also recall the following notions.

Let $Q$ be a probability measure on $T$. Assume that $M$ is a probability on the $\sigma$-algebra $\Sigma \otimes T$ such that $P$ and $Q$ are the marginals of $M$. Assume also that for each $y \in \Upsilon$ there exists a probability $P_{y}$ on $\Sigma$, satisfying the following properties:

(D1) For every $A \in \Sigma$ the map $y \mapsto P_{y}(A)$ is $T$-measurable;

(D2) $M(A \times B)=\int_{B} P_{y}(A) Q(\mathrm{~d} y)$ for each $A \times B \in \Sigma \otimes T$. 
Then, $\left\{P_{y}\right\}_{y \in \Upsilon}$ is said to be a product regular conditional probability (product rcp for short) on $\Sigma$ for $M$ with respect to $Q$ (see [5: Section 2] or [23: Definition 1.1]).

Let $I$ be an arbitrary non-empty index set. If $\left\{\left(\Omega_{i}, \Sigma_{i}, P_{i}\right)\right\}_{i \in I}$ is a family of probability spaces then, for each $\emptyset \neq J \subseteq I$, we denote by $\left(\Omega_{J}, \Sigma_{J}, P_{J}\right)$ the product probability space $\otimes_{i \in J}\left(\Omega_{i}, \Sigma_{i}, P_{i}\right):=\left(\prod_{i \in J} \Omega_{i}, \otimes_{i \in J} \Sigma_{i}, \otimes_{i \in J} P_{i}\right)$. If $(\Omega, \Sigma, P)$ is a probability space, we write $P_{I}$ for the product measure on $\Omega^{I}$ and $\Sigma_{I}$ for its domain.

LEMMA 4.1. Let $\mathcal{F}$ be a $\sigma$-subalgebra of $\Sigma$ and let $\left\{X_{i}\right\}_{i \in I}$ be a non empty family of $\Sigma$-T-measurable maps from $\Omega$ into $\Upsilon$ such that $\left\{X_{i}\right\}_{i \in I}$ is $P$-conditionally i.i.d. over $\mathcal{F}$. Suppose that $T$ is countably generated and that $P_{X_{i}}$ is perfect for each $i \in I$. Then there exists a probability measure $M$ on $T \otimes \mathcal{F}$ with marginal $R:=P \nmid \mathcal{F}$ such that $M:=P \circ\left(X_{i} \times \operatorname{id}_{\Omega}\right)^{-1}$ for every $i \in I$, and a product $r c p\left\{Q_{\omega}\right\}_{\omega \in \Omega}$ on $T$ for $M$ with respect to $R$, such that

(i) for any fixed $B \in T$ and $i \in I$ the $\operatorname{map} Q .(B): \Omega \rightarrow[0,1]$ is $R$-a.s. equal to $P\left(X_{i}^{-1}(B) \mid \mathcal{F}\right)(\cdot)$;

(ii) $\int_{F} Q_{\omega}^{I}(H) R(\mathrm{~d} \omega)=P\left(F \cap X^{-1}(H)\right)$ for every $F \in \mathcal{F}$ and $H \in T_{I}$, where $Q_{\omega}^{I}$ denotes the $I$-fold product probability $\otimes_{i \in I} P_{i}$ of copies $P_{i}:=Q_{\omega}$ of $Q_{\omega}$ for $i \in I$, and $X: \Omega \rightarrow \Upsilon^{I}$ is defined by $X(\omega)=\left(X_{i}(\omega)\right)_{i \in I}$ for each $\omega \in \Omega$.

Proof. First fix on an arbitrary $i \in I$.

(a) The function $X_{i} \times \operatorname{id}_{\Omega}$ from $\Omega$ into $\Upsilon \times \Omega$ defined by means of

$$
\left(X_{i} \times \operatorname{id}_{\Omega}\right)(\omega):=\left(X_{i}(\omega), \omega\right) \text { for each } \omega \in \Omega
$$

is $\Sigma$-T $\otimes \mathcal{F}$-measurable. So, we have a probability measure $M_{i}:=P \circ\left(X_{i} \times \operatorname{id}_{\Omega}\right)^{-1}$ on $T \otimes \mathcal{F}$. Since all $X_{i}$ are $P$-conditionally identically distributed over $\mathcal{F}$, it follows that $M_{i}$ is independent of $i$, so we may write $M:=M_{i^{*}}$ for any fixed $i^{*} \in I$.

(b) There exists a product $\operatorname{rcp}\left\{Q_{\omega}\right\}_{\omega \in \Omega}$ on $T$ for $M$ with respect to $R=P \uparrow \mathcal{F}$ such that for any fixed $B \in T$

$$
Q \bullet(B)=P\left(X_{i}^{-1}(B) \mid \mathcal{F}\right)(\cdot) \quad R-\text { a.s.. }
$$

In fact, by assumption each marginal measure $P_{X_{i}}$ of $M$ on $T$ is perfect and $T$ is countably generated; hence by [5. Theorem 6], there exists a product $\operatorname{rcp}\left\{Q_{\omega}\right\}_{\omega \in \Omega}$ on $T$ for $M$ with respect to $R$.

Since $\left\{Q_{\omega}\right\}_{\omega \in \Omega}$ satisfies (D2), we get that

$$
\int_{F} Q_{\omega}(B) R(\mathrm{~d} \omega)=M(B \times F)=P\left(F \cap X_{i}^{-1}(B)\right)=\int_{F} P\left(X_{i}^{-1}(B) \mid \mathcal{F}\right)(\omega) R(\mathrm{~d} \omega)
$$

for every $B \in T$ and $F \in \mathcal{F}$, which proves (b); hence (i) follows.

(c) Using (i) and a monotone class argument, we get that (ii) holds true.

The next result extends a corresponding one due to Olshen (see 18: Theorem (3)]) concerning a generalization of de Finetti's Theorem.

Proposition 4.1. Let $\left\{X_{i}\right\}_{i \in I}$ be a P-exchangeable infinite family of $\Sigma$-T-measurable maps from $\Omega$ into $\Upsilon$. Suppose that $T$ is countably generated and $P_{X_{i}}$ is perfect for each $i \in I$. Then there exists a d-dimensional random vector $\Theta$ such that $\left\{X_{i}\right\}_{i \in I}$ is $P$-conditionally i.i.d. given $\Theta$.

Proof. Since $\left\{X_{i}\right\}_{i \in I}$ is $P$-exchangeable, it follows by [7: Theorem 459B], that there exist a $\sigma$-subalgebra $\mathcal{F}$ of $\Sigma$ such that $\left\{X_{i}\right\}_{i \in I}$ is $P$-conditionally i.i.d. over $\mathcal{F}$. So, applying Lemma 4.1 . 


\section{SOME CHARACTERIZATIONS OF MRPS}

we deduce that there exists a family $\left\{Q_{\omega}\right\}_{\omega \in \Omega}$ of $\mathcal{F}$-T-Markov kernels such that

$$
\int_{F} Q_{\omega}^{I}(H) R(\mathrm{~d} \omega)=P\left(F \cap X^{-1}(H)\right)
$$

for every $H \in T_{I}$ and $F \in \mathcal{F}$, where $R:=P \mid \mathcal{F}$. Then there exists a countably generated $\sigma$-subalgebra $\mathcal{A}$ of $\mathcal{F}$ such that $Q_{\bullet}(B)$ is $\mathcal{A}$-measurable for arbitrary but fixed $B \in T$ (take, e.g., $\mathcal{A}_{B}:=\left\{[Q \bullet(B)]^{-1}(E): E \in \mathcal{G}_{\mathfrak{B}}\right\}$ for $B \in T$, and $\mathcal{A}:=\sigma\left(\bigcup_{B \in \mathcal{G}_{T}} \mathcal{A}_{B}\right)$, where $\mathcal{G}_{\mathfrak{B}}$ and $\mathcal{G}_{T}$ is a countable generator of $\mathfrak{B}$ and $T$, respectively). Since $\mathcal{A}$ is countably generated, there exists a map $\widetilde{\Theta}: \Omega \rightarrow \mathbb{R}$ such that $\mathcal{A}=\sigma(\widetilde{\Theta})$ (take, e.g., $\widetilde{\Theta}$ to be the Marczewski functional on $\Omega$, cf. e.g., 6 . $343 \mathrm{E}]$ for the definition). But since $\left\{X_{i}\right\}_{i \in I}$ is $P$-conditionally i.i.d. over $\mathcal{F}$ and $\mathcal{A} \subseteq \mathcal{F}$, it follows that $\left\{X_{i}\right\}_{i \in I}$ is so over $\mathcal{A}=\sigma(\widetilde{\Theta})$.

Note also that since $\mathbb{R}$ and $\mathbb{R}^{d}$ are standard Borel spaces of the same cardinality, there exists a Borel isomorphism $g$ from $\mathbb{R}$ into $\mathbb{R}^{d}$ (cf. e.g., 7 : Corollary $\left.424 \mathrm{D}(\mathrm{a})\right]$ ). So, putting $\Theta:=g \circ \widetilde{\Theta}$, we get that $\Theta$ is a $d$-dimensional random vector on $\Omega$ such that $\sigma(\Theta)=\sigma(\widetilde{\Theta})$.

Corollary 4.1.1 (see R. Olshen, [18. Theorem 3]). If $\left\{X_{n}\right\}_{\in \mathbb{N}}$ is a P-exchangeable sequence of measurable maps from $\Omega$ into a complete, separable metric space, then there exists a real-valued random variable $\Theta$ on $\Omega$ such that $\left\{X_{n}\right\}_{n \in \mathbb{N}}$ is $P$-conditionally i.i.d. given $\Theta$.

THEOREM 4.2. Let $\left\{X_{i}\right\}_{i \in I}$ be an infinite family of $\Sigma$-T-measurable maps from $\Omega$ into $\Upsilon$. Consider the following assertions:

(i) $\left\{X_{i}\right\}_{i \in I}$ is P-exchangeable.

(ii) There exists a $\sigma$-subalgebra $\mathcal{F}$ of $\Sigma$ such that $\left\{X_{i}\right\}_{i \in I}$ is $P$-conditionally i.i.d. over $\mathcal{F}$.

(iii) There exists a $\sigma$-subalgebra $\mathcal{F}$ of $\Sigma$ and a family $\left\{Q_{\omega}\right\}_{\omega \in \Omega}$ of $\mathcal{F}$-T-Markov kernels such that

$$
\int_{F} Q_{\omega}^{I}(H) R(\mathrm{~d} \omega)=P\left(F \cap X^{-1}(H)\right)
$$

for every $H \in T_{I}$ and $F \in \mathcal{F}$, where $R:=P \uparrow \mathcal{F}$ and $Q_{\omega}^{I}, X$ are as in Lemma 4.1.

(iv) There exists a $\Sigma$ - $\mathfrak{B}_{d}$-measurable map $\Theta$ from $\Omega$ into $\mathbb{R}^{d}$ such that $\left\{X_{i}\right\}_{i \in I}$ is $P$ - conditionally i.i.d. given $\Theta$.

Then (iii) $\Longrightarrow$ (i) $\Longleftrightarrow$ (ii) and (iv) $\Longrightarrow$ (i). If any one of conditions (i) to (iv) is satisfied, then all image measures $P_{X_{i}}$ are equal.

Moreover, if $P_{X_{i}}$ is perfect for any $i \in I$ and $T$ is countably generated, then assertions (i) to (iv) are all equivalent.

Pr o of. The equivalence (i) $\Longleftrightarrow$ (ii) follows by $[7$. Theorem 459B], while the implications (iii) $\Longrightarrow(\mathrm{i})$ and (iv) $\Longrightarrow$ (i) are evident.

Clearly, if assertion (i) or equivalently (ii) is satisfied then all $P_{X_{i}}$ are equal and the same applies if (iii) or (iv) holds true.

Moreover, if every measure $P_{X_{i}}$ is perfect and $T$ is countably generated, then implications (ii) $\Longrightarrow$ (iii) and (i) $\Longrightarrow$ (iv) follow from Lemma 4.1 and Proposition 4.1 respectively. So, we get that assertions (i) to (iv) are all equivalent.

CorollarY 4.2.1. Let $\left\{X_{t}\right\}_{t \in \mathbb{R}_{+}}$be a family of $\Sigma$-T-measurable maps from $\Omega$ into $\Upsilon$. Suppose that $\Sigma$ is countably generated, $P$ is perfect, $\Upsilon$ is a Polish space, $\left\{X_{t}\right\}_{t \in \mathbb{R}_{+}}$is separable with separator $\mathbb{Q}_{+}$and that its canonical filtration is right-continuous. Then each of the items (i) to (iv) of Theorem 4.2 is equivalent to condition 
(v) there exist a d-dimensional random vector $\Theta$ and an $r c p\left\{P_{\theta}\right\}_{\theta \in \mathbb{R}^{d}}$ of $P$ over $P_{\Theta}$ consistent with $\Theta$ such that $\left\{X_{t}\right\}_{t \in \mathbb{R}_{+}}$is $P_{\theta}$-i.i.d. for $P_{\Theta}$-a.a. $\theta \in \mathbb{R}^{d}$.

P r o o f. It follows by our assumptions for $P, \Sigma$ and $\Upsilon$, that given a $d$-dimensional random vector $\Theta$, there exists an $\operatorname{rcp}\left\{P_{\theta}\right\}_{\theta \in \mathbb{R}^{d}}$ of $P$ over $P_{\Theta}$ consistent with $\Theta$. Thus, we may apply Proposition 3.2 to obtain that condition (v) is equivalent to (iv) of Theorem 4.2 . The equivalence of all items (i) to $(\mathrm{v})$ is immediate by Theorem 4.2 .

Remarks 3. (a) The assumption " $P_{X_{i}}$ perfect" made in the last theorem is easily verified in the usual applications, since this is covered by the following facts: $(\alpha)$ If $\Upsilon$ is a Polish space then each $P_{X_{i}}$ is Radon (cf. e.g., [7: Proposition $\left.434 \mathrm{~K}(\mathrm{~b})\right]$ and 7?: Definition $411 \mathrm{H}(\mathrm{b})$ ] for the definition of a Radon measure); hence perfect (cf. e.g., 7: Proposition 416W(a)]). ( $\beta$ ) If $P$ is perfect then each $P_{X_{i}}$ is so (cf. e.g., 7. Proposition $\left.\left.451 \mathrm{E}(\mathrm{a})\right]\right)$. ( $(\gamma)$ If $\Omega=\Upsilon^{I}, X_{i}(i \in I)$ are the canonical projections from $\Omega$ onto $\Upsilon$, and $P$ is any probability measure on $\Sigma:=T_{I}$ then each $P_{X_{i}}$ is perfect if and only if $P$ is perfect (cf. e.g., [7: Theorem $454 \mathrm{~A}(\mathrm{~b})(\mathrm{iii})]$ ).

(b) To the best of our knowledge, the most general result concerning the equivalence of assertions (i) and (iii) of Theorem 4.2 is Theorem 1.1 from [12, which extends de Finetti's Theorem, saying that for each infinite sequence $\left\{X_{n}\right\}_{n \in \mathbb{N}}$ of random variables taking values in a standard Borel space $\Upsilon$ (i.e. $\Upsilon$ is isomorphic to some Borel-measurable subset of $\mathbb{R}$ ) assertions (i) and (iii) of Theorem 4.2 with $\left\{X_{n}\right\}_{n \in \mathbb{N}}$ in the place of $\left\{X_{i}\right\}_{i \in I}$ are equivalent. It is well-known that any Polish space is standard Borel; in particular, $\mathbb{R}^{d}$ and $\mathbb{R}^{\mathbb{N}}$ are such spaces.

(c) There are measurable spaces $(\Upsilon, T)$ satisfying the assumptions of Theorem 4.2 , i.e., that $T$ is countably generated and each $P_{X_{i}}$ is perfect, which are not standard Borel spaces; hence Theorem 4.2 extends Theorem 1.1 from 12 . In fact, it is known that each uncountable analytic Hausdorff space (i.e., a non-empty topological Hausdorff space being a continuous image of the space $\mathbb{N}^{\mathbb{N}}$, cf. e.g., 77. Definition 423A]) has a non-Borel analytic subset (cf. e.g., 7. Proposition 423L]). It is also known that for each analytic Hausdorff space $\Upsilon$ the Borel $\sigma$-algebra $\mathfrak{B}(\Upsilon)$ is countably generated (cf. e.g., [7; 423X(d)]), and that any Borel probability measure on $\mathfrak{B}(\Upsilon$ ) is always inner regular with respect to compact sets (see [10 Chapter IV, Theorem 1, p. 195]); hence it is perfect (cf. e.g., 7. Proposition 451C]). Consequently, each uncountable analytic Hausdorff space has a subset satisfying the assumptions of Theorem 4.2 but not being a standard Borel space.

(d) Restricting attention to measurable spaces $(\Upsilon, T)$ satisfying the countability assumption of $T$ as in Theorem 4.2, costs us some generality; for instance, the general compact Hausdorff space does not satisfy the countability assumption for $T$, and it is known that the equivalence of assertions (i) to (iii) of Theorem 4.2 is true for countable products of compact Hausdorff spaces (see [9] or [3]). More general, the equivalence of assertions (i) to (iii) of Theorem 4.2 is proven in 7 . Theorem $459 \mathrm{G}]$ for uncountable products of general Hausdorff spaces. But all the above are specialized in the product situation of topological spaces, while assertions (i) to (iii) of Theorem 4.2 have the advantage of being free from any topological assumption as well as from any product situation.

The following definition of an MRP traces back to Huang [11. Section 1, Definition 3].

Definition 4.1. The counting process $\left\{N_{t}\right\}_{t \in \mathbb{R}_{+}}$is said to be a $\nu$-mixed renewal process associated with $\left\{P_{\widetilde{y}}\right\}_{\widetilde{y} \in \widetilde{r}}$, if for every $r \in \mathbb{N}$ and for all $w_{1}, \ldots, w_{r} \in \mathbb{R}$ condition

$$
P\left(\bigcap_{k=1}^{r}\left\{W_{k} \leq w_{k}\right\}\right)=\int \prod_{k=1}^{r} P_{\widetilde{y}}\left(W_{k} \leq w_{k}\right) \nu(\mathrm{d} \widetilde{y}),
$$


holds true, where $\left\{P_{\widetilde{y}}\right\}_{\widetilde{y} \in \widetilde{\Upsilon}}$ is a family of probability measures on $\Sigma$ and $\nu$ is a probability measure on $B(\widetilde{\Upsilon}):=\sigma\left(\left\{P_{\bullet}(E): E \in \Sigma\right\}\right)$ such that for $\nu$-a.a. $\widetilde{y} \in \widetilde{\Upsilon}$ the process $\left\{W_{n}\right\}_{n \in \mathbb{N}}$ is $P_{\widetilde{y}}$-identically distributed.

In Huang's definition it is assumed that $\left\{N_{t}\right\}_{t \in \mathbb{R}_{+}}$takes values only in $\mathbb{N}_{0}$, which is equivalent to the mild assumption that $\left\{N_{t}\right\}_{t \in \mathbb{R}_{+}}$has zero probability of explosion, that is $P\left(\bigcup_{t \in(0, \infty)}\left\{N_{t}=\infty\right\}\right)=0$ (cf. e.g., 21: Lemma 2.1.4]).

Remark 4. Note that in Huang's 11] definition the assumption that $\left\{W_{n}\right\}_{n \in \mathbb{N}}$ is $P_{\widetilde{y}}$-identically distributed for $\nu$-a.a. $\widetilde{y} \in \widetilde{\Upsilon}$ is not written explicitly. But this assumption must be included there, since it is necessary for the validity of the Corollary in page 20 of 11, as it follows from Example 5 below.

In fact, consider the process $\left\{N_{t}\right\}_{t \in \mathbb{R}_{+}}$of Example 5 , where the above assumption does not hold true, as well as Huang's definition of a $\nu$-MRP without the above assumption. Also note that $q:=P(Z<\infty)=0<1$, where $Z$ is the almost sure limit of $\left\{N_{t}\right\}_{t \in \mathbb{R}_{+}}$as $t \rightarrow \infty$. Assume, if possible, that the Corollary in [11 p. 20] holds true. Then conditional on the event $\{Z=\infty\}$ the process $\left\{N_{t}\right\}_{t \in \mathbb{R}_{+}}$has the exchangeable property $(E)$ (see 11: Definition 1] for the definition) implying that $\left\{W_{n}\right\}_{n \in \mathbb{N}}$ is exchangeable, a contradiction to Example 5 .

TheOREM 4.3. Consider the following assertions:

(i) There exists a d-dimensional $(d \in \mathbb{N})$ random vector $\Theta$ such that $\left\{N_{t}\right\}_{t \in \mathbb{R}_{+}}$is a $(P, \mathbf{K}(\Theta))$ $M R P$.

(ii) There exist a random vector $\Theta$, an $r c p\left\{P_{\theta}\right\}_{\theta \in \mathbb{R}^{d}}$ of $P$ over $P_{\Theta}$ consistent with $\Theta$, and a family $\{\mathbf{K}(\theta)\}_{\theta \in \mathbb{R}^{d}}$ of Z-B-Markov kernels for $P_{\Theta-\text { a.a. }} \theta \in \mathbb{R}^{d}$ such that the family $\left\{N_{t}\right\}_{t \in \mathbb{R}_{+}}$is a $\left(P_{\theta}, \mathbf{K}(\theta)\right)-R P$.

(iii) The process $\left\{W_{n}\right\}_{n \in \mathbb{N}}$ is $P$-exchangeable.

(iv) There exist a $\sigma$-subalgebra $\mathcal{F}$ of $\Sigma$ and a family $\left\{Q_{\omega}\right\}_{\omega \in \Omega}$ of $\mathcal{F}-\mathfrak{B}$-Markov kernels such that

$$
\int_{F} Q_{\omega}^{\mathbb{N}}(H) R(\mathrm{~d} \omega)=P\left(F \cap W^{-1}(H)\right)
$$

for every $H \in \mathfrak{B}_{\mathbb{N}}$ and $F \in \mathcal{F}$, where $R:=P \uparrow \mathcal{F}, W:=\left(W_{1}, \ldots, W_{n}, \ldots\right)$ and $Q_{\omega}^{\mathbb{N}}$ denotes the $\mathbb{N}$-fold product probability $\otimes_{n \in \mathbb{N}} P_{n}$ of copies $P_{n}:=Q_{\omega}$ of $Q_{\omega}$ for $n \in \mathbb{N}$.

(v) There exist a $\sigma$-subalgebra $\mathcal{F}$ of $\Sigma$, a subfield $\operatorname{rcp}\left\{S_{\omega}\right\}_{\omega \in \Omega}$ for $P$ over the restriction $R:=$ $P \nmid \mathcal{F}$, and a family $\{\mathbf{K}(\omega)\}_{\omega \in \Omega}$ of $\mathcal{F}-\mathfrak{B}$-Markov kernels such that for $R$-a.a. $\omega \in \Omega$ the sequence $\left\{W_{n}\right\}_{n \in \mathbb{N}}$ is $S_{\omega}$-independent and condition $\left(S_{\omega}\right)_{W_{n}}=\mathbf{K}(\omega)$ holds for each $n \in \mathbb{N}$.

(vi) There exist a set $\widetilde{\Upsilon}$, a family $\left\{S_{\widetilde{y}}\right\}_{\widetilde{y} \in \widetilde{\Upsilon}}$ of probability measures on $\Sigma$ and a probability measure $\nu$ on $B(\widetilde{\Upsilon}):=\sigma\left(\left\{S_{\bullet}(E): E \in \Sigma\right\}\right)$ such that $\left\{N_{t}\right\}_{t \in \mathbb{R}_{+}}$is a $\nu$-MRP associated with $\left\{S_{\widetilde{y}}\right\}_{\widetilde{y} \in \Upsilon}$.

Then the following implications hold true:

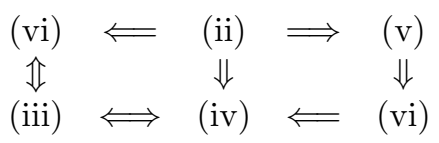

Moreover, if $\Sigma$ is countably generated and $P$ is perfect then items (i) to (vi) are all equivalent.

Proof. First note that the implications (i) $\Longrightarrow$ (iii) and (vi) $\Longrightarrow$ (iii) are obvious. The implication (ii) $\Longrightarrow$ (i) is immediate by Proposition 3.1 , while the implication (iii) $\Longrightarrow$ (i) and the equivalence 
of (iii) and (iv) follow directly by Proposition 4.1 and Theorem 4.2 , respectively, since for $(\Upsilon, T)=$ $(\mathbb{R}, \mathfrak{B})$ every measure $P_{W_{n}}$ on $\mathfrak{B}$ is perfect and $\mathfrak{B}$ is countably generated. The latter equivalence together with implication (vi) $\Longrightarrow$ (iii) yields (vi) $\Longrightarrow$ (iv).

Ad (ii) $\Longrightarrow$ (iv): If (ii) holds true, then there exists a $P_{\Theta}$-null set $H_{*} \in \mathfrak{B}_{d}$ such that for any $\theta \notin H_{*}$ the process $\left\{W_{n}\right\}_{n \in \mathbb{N}}$ is $P_{\theta}$-exchangeable, implying together with property (d2) its $P$-exchangeability as well; hence (iii) or equivalently (iv) follows.

Ad (ii) $\Longrightarrow(\mathrm{v})$ : Assume that (ii) is true. Putting $S_{\omega}(E):=P_{\theta}(E)$ for any $\omega \in \Omega, E \in \Sigma$ and $\theta=\Theta(\omega)$, we clearly get that $\left\{S_{\omega}\right\}_{\omega \in \Omega}$ is a subfield $\operatorname{rcp}$ for $P$ over $R:=P \uparrow \sigma(\Theta)$ such that the interarrival times $W_{n}, n \in \mathbb{N}$, are $S_{\omega}$-i.i.d. with a common probability distribution $\mathbf{K}(\omega)$ for any $\omega \notin H_{* *}:=\Theta^{-1}\left(H_{*}\right)$, where $\mathbf{K}(\omega):=\mathbf{K}(\theta)$ for each $\omega \in \Omega$ and $\Theta(\omega)=\theta \notin H_{*} \in \mathfrak{B}_{d}$. Since clearly $H_{* *}$ is an $R$-null set, it follows that $\left\{S_{\omega}\right\}_{\omega \in \Omega}, \mathcal{F}:=\sigma(\Theta)$ and $\{\mathbf{K}(\omega)\}_{\omega \in \Omega}$ satisfy assertion (v).

Ad (v) $\Longrightarrow$ (vi): Assume that (v) holds true and let $\mathcal{F},\left\{S_{\omega}\right\}_{\omega \in \Omega}$ and $R$ be as in (v). Put $\widetilde{\Upsilon}:=\Omega,\left\{S_{\widetilde{y}}\right\}_{\widetilde{y} \in \widetilde{\Upsilon}}:=\left\{S_{\omega}\right\}_{\omega \in \Omega}$ and $B(\widetilde{\Upsilon}):=\sigma\left(\left\{S_{\bullet}(E): E \in \Sigma\right\}\right)$. Then $B(\widetilde{\Upsilon}) \subseteq \mathcal{F}$ and so, we may define the probability measure $\nu:=R \uparrow B(\widetilde{\Upsilon})$. Since by (v) the process $\left\{W_{n}\right\}_{n \in \mathbb{N}}$ is $S_{\omega}$-i.i.d. for $R$-a.a. $\omega \in \Omega$, we get that it is $S_{\widetilde{y}}$-i.i.d. for $\nu$-a.a. $\widetilde{y} \in \widetilde{\Upsilon}$. The latter together with the definition of a subfield rcp yields that $\left\{N_{t}\right\}_{t \in \mathbb{R}_{+}}$is a $\nu$-MRP associated with $\left\{S_{\widetilde{y}}\right\}_{\widetilde{y} \in \Upsilon}$; hence assertion (vi) follows.

Moreover, if $\Sigma$ is countably generated and $P$ is perfect, the implication (iv) $\Longrightarrow(\mathrm{v}$ ) holds true. In fact, by Theorem 4.2 , we obtain that assertion (iv) is equivalent with the fact that $\left\{W_{n}\right\}_{n \in \mathbb{N}}$ is $P$-conditionally i.i.d. over $\mathcal{F}$. But since $\Sigma$ is countably generated and $P$ is perfect, there exists a subfield $\operatorname{rcp}\left\{S_{\omega}\right\}_{\omega \in \Omega}$ for $P$ over $R:=P\lceil\mathcal{F}$. Thus, we may apply Lemma 3.4(ii) for $(\Psi, Z):=(\Omega, \mathcal{F})$ and $\Theta:=\mathrm{id}_{\Omega}$ to get $(\mathrm{v})$.

Assuming now that (i) holds true, it follows again by our assumptions for $\Sigma$ and $P$ that there exists an $\operatorname{rcp}\left\{P_{\theta}\right\}_{\theta \in \mathbb{R}^{d}}$ of $P$ over $P_{\Theta}$ consistent with $\Theta$. So according to Proposition 3.1. we get that for $P_{\Theta}$-a.a. $\theta \in \mathbb{R}^{d}$ the family $\left\{N_{t}\right\}_{t \in \mathbb{R}_{+}}$is a $\left(P_{\theta}, \mathbf{K}(\theta)\right)$-RP; hence (i) implies (ii).

Thus, assuming that $\Sigma$ is countably generated and $P$ is perfect, we obtain that items (i) to (vi) are all equivalent. This completes the whole proof.

Note that the most important applications in Probability Theory are still rooted in the case of standard Borel spaces; hence of spaces satisfying always the assumptions of the above theorem concerning $P$ and $\Sigma$.

\section{Examples and applications}

In this section, we provide two groups of examples: The first one (Examples 1 to 2 shows that the perfectness assumption for the probability measures as well as the countability assumption for the $\sigma$-algebras involved in Theorems $4.2,4.3$ and in Corollary 4.2.1. are essential for the validity of the equivalences obtained therein.

In the second group of examples (Examples 3 to 5 ), the existence of non-trivial probability spaces admitting $(P, \mathbf{K}(\Theta))$-MRPs with prescribed distributions for their interarrival processes as well as for the parameter $\Theta$ is proven, providing in this way a method of constructing such processes. As a consequence, concrete examples of MRPs are presented. It is also worth noticing that our construction relies on Proposition 3.1 and allows us to obtain probability spaces that satisfy all assumptions of Theorems 4.2 and 4.3 . 
It follows an example to show that the perfectness assumption for the probability measures $P_{X_{i}}$ in Theorem 4.2 and its Corollary 4.2.1 is essential for the validity of the equivalences of (i) to (v).

Example 1. Let $\Upsilon:=\mathbb{R}_{+}$and let $\widetilde{Q}$ be a probability measure on $\mathfrak{B}(\Upsilon)$. Consider a subset $B$ of $\Upsilon$ such that $\widetilde{Q}^{*}(B)=\widetilde{Q}^{*}\left(B^{c}\right)=1$, where $\widetilde{Q}^{*}$ is the outer measure induced by $\widetilde{Q}$. Let $T:=\sigma(\mathfrak{B}(\Upsilon), B)$ and let $Q: T \rightarrow[0,1]$ be the probability measure defined by

$$
Q(A):=\widetilde{Q}^{*}(A \cap B) \quad \text { for each } A \in T .
$$

Then $Q$ is non perfect. Set $\Omega:=\Upsilon^{\mathbb{N}}, \Sigma:=T_{\mathbb{N}}, P:=Q^{\mathbb{N}}, W_{n}:=\pi_{n}: \Omega \rightarrow \Upsilon$, where $\pi_{n}$ is the canonical projection for any $n \in \mathbb{N}$. Clearly, assertion (i) of Theorem 4.2 is satisfied by $\left\{W_{n}\right\}_{n \in \mathbb{N}}$; hence by Theorem 4.2 , we equivalently get that (ii) holds true.

Assume that assertion (iii) of Theorem 4.2 is valid, i.e. that there exists a $\sigma$-subalgebra $\mathcal{F}$ of $\Sigma$ and a family $\left\{Q_{\omega}\right\}_{\omega \in \Omega}$ of $\mathcal{F}$-T-Markov kernels such that

$$
\int_{F} Q_{\omega}^{\mathbb{N}}(H) R(\mathrm{~d} \omega)=P\left(F \cap W^{-1}(H)\right)
$$

for every $H \in T_{\mathbb{N}}$ and $F \in \mathcal{F}$, where $R:=P\left\lceil\mathcal{F}\right.$ and $Q_{\omega}^{\mathbb{N}}, W$ are as in Theorem 4.2. Then $\left\{Q_{\omega}^{\mathbb{N}}\right\}_{\omega \in \Omega}$ is a subfield $\operatorname{rcp}$ for $P$ over $R$; hence, we may and do assume that $\mathcal{F}$ is countably generated. Applying now a monotone class argument we deduce that there exists an $R$-null set $N \in \mathcal{F}$ such that for each $A \in \mathcal{F}$ condition $Q_{\omega}^{\mathbb{N}}(A)=1$ holds true for any $\omega \in N^{c} \cap A$.

But since $\left\{Q_{\omega}^{\mathbb{N}}\right\}_{\omega \in \Omega}$ is a subfield rcp, we get for every $F \in \mathcal{F}$ that

$$
\int_{F} Q_{\omega}^{\mathbb{N}}\left(B^{\mathbb{N}}\right) R(\mathrm{~d} \omega)=P\left(F \cap B^{\mathbb{N}}\right)=P(F)=\int_{F} \chi_{F} \mathrm{~d} R,
$$

implying that $P(D)=0$, where $D:=\left\{\omega \in \Omega: Q_{\omega}^{\mathbb{N}}\left(B^{\mathbb{N}}\right) \neq 1\right\}$.

Put $E:=D \cup N$. For any $\omega \in E^{c}$, we get $Q_{\omega}^{\mathbb{N}}(\{\omega\})=1$ and $Q_{\omega}^{\mathbb{N}}\left(B^{\mathbb{N}}\right)=1$; hence $Q_{\omega}^{\mathbb{N}}\left(B^{\mathbb{N}} \cap\{\omega\}\right)=$ 1 , implying $B^{\mathbb{N}} \cap\{\omega\} \neq \emptyset$ or $\omega \in B^{\mathbb{N}}$. Thus, we get $E^{c} \subseteq B^{\mathbb{N}}$. But then $\widetilde{Q}^{*}\left(B^{c}\right)=1$ yields $P\left(E^{c}\right)=0$ or equivalently $P(E)=1$, a contradiction.

Assume now that assertion (v) of Corollary 4.2 .1 is valid, i.e., that there exist a $d$-dimensional random vector $\Theta$ and an $\operatorname{rcp}\left\{P_{\theta}\right\}_{\theta \in \mathbb{R}^{d}}$ of $P$ over $P_{\Theta}$ consistent with $\Theta$ such that $\left\{W_{n}\right\}_{n \in \mathbb{N}}$ is $P_{\theta}$-i.i.d. for $P_{\Theta}$-a.a. $\theta \in \mathbb{R}^{d}$. For any fixed $A \in \Sigma$ define $\widetilde{R} \bullet(A): \Omega \rightarrow[0,1]$ by $\widetilde{R}_{\omega}(A):=(P \bullet(A) \circ \Theta)(\omega)$. Then $\left\{\widetilde{R}_{\omega}\right\}_{\omega \in \Omega}$ is a subfield rcp for $P$ over $P \uparrow \sigma(\Theta)$, a contradiction according to the above arguments.

It follows an example to show that the countability assumption for $T$ or $\Sigma$ in Corollary 4.2 .1 is essential for the validity of the equivalences of (i) to (v) in Theorem 4.2 and Corollary 4.2.1

Example 2. Let $\Upsilon:=\mathbb{R}_{+}$, let $\widetilde{Q}$ be a probability measure on $\mathfrak{B}(\Upsilon)$, let $T$ be the completion of $\mathfrak{B}(\Upsilon)$ with respect to $\widetilde{Q}$, and let $Q$ be the completion of $\widetilde{Q}$. Put $\Omega:=\Upsilon^{\mathbb{N}}, \widetilde{\Sigma}:=T_{\mathbb{N}}$ and $\widetilde{P}:=Q^{\mathbb{N}}$. Denote by $\Sigma$ the completion of $\widetilde{\Sigma}$ with respect to $\widetilde{P}$ and by $P$ the completion of $\widetilde{P}$. Then $P$ is a perfect probability measure on $\Sigma$ (cf. e.g., 7. Proposition $451 \mathrm{G}$ and Theorem $451 \mathrm{~J}]$ ) but $\Sigma$ and $T$ are not countably generated.

Put $W_{n}:=\pi_{n}: \Omega \rightarrow \Upsilon$ for any $n \in \mathbb{N}$. Clearly, $\left\{W_{n}\right\}_{n \in \mathbb{N}}$ is $P$-exchangable, that is, assertion (i) of Theorem 4.2 is valid; hence assertion (ii) of the same theorem is also valid.

Assume now that assertion (v) of Corollary 4.2 .1 is valid. It then follows that there exists a $d$-dimensional random vector $\Theta$ and an $\operatorname{rcp}\left\{P_{\theta}\right\}_{\theta \in \mathbb{R}^{d}}$ of $P$ over $P_{\Theta}$ consistent with $\Theta$, implying the existence of a subfiled $\operatorname{rcp}\left\{R_{\omega}\right\}_{\omega \in \Omega}$ of $P$ over $P\left\lceil\sigma(\Theta)\right.$, where each function $R_{\bullet}(A): \Omega \rightarrow[0,1]$ is defined by $R_{\omega}(A):=(P \bullet(A) \circ \Theta)(\omega)$ for any fixed $A \in \Sigma$. Since $\sigma(\Theta)$ is countably generated, it 
follows as in Example 1 that there exists a set $N \in \sigma(\Theta)$ such that $P(N)=0$ and for any $A \in \sigma(\Theta)$ condition $R_{\omega}(A)=1$ holds true for any $\omega \in N^{c} \cap A$.

Choose a set $D \subseteq N^{c}$ such that $D \notin \sigma(\Theta)$ but $D \in \Sigma$. Then for each $\omega \notin N$, we obtain

$$
1=R_{\omega}(\{\omega\}) \leq R_{\omega}(D) \leq 1 \quad \text { if } \omega \in D
$$

and

$$
1=R_{\omega}(\{\omega\}) \leq R_{\omega}\left(D^{c}\right) \leq 1 \quad \text { if } \omega \in D^{c} .
$$

Thus, $D=N^{c} \cap\left\{\omega \in \Omega: R_{\omega}(D)=1\right\} \in \sigma(\Theta)$, which is impossible by the choice of $D$; hence assertion (v) of Corollary 4.2 .1 is not valid.

The next counterexample shows that there exists a non perfect probability space $(\Omega, \Sigma, P)$ and a counting process $\left\{N_{t}\right\}_{t \in \mathbb{R}_{+}}$on it satisfying conditions (i), (iii), (iv) and (vi) of Theorem 4.3 but not (ii) and (v); hence the perfectness of $P$ is an essential assumption.

Counterexample 1. Let $\Upsilon:=\mathbb{R}_{+}$, let $Q$ be a probability measure on $T:=\mathfrak{B}(\Upsilon)$, and let $(\Omega, \widetilde{\Sigma}, \widetilde{P}):=\left(\Upsilon^{\mathbb{N}}, T_{\mathbb{N}}, Q^{\mathbb{N}}\right)$. Consider a subset $B$ of $\Upsilon^{\mathbb{N}}$ such that $\widetilde{P}^{*}(B)=\widetilde{P}^{*}\left(B^{c}\right)=1$, the $\sigma$ algebra $\Sigma:=\sigma(\{\widetilde{\Sigma} \cup B\})$ and the non perfect probability measure $P$ on $\Sigma$ defined by means of

$$
P(A):=\widetilde{P}^{*}(A \cap B) \text { for each } A \in \Sigma .
$$

Let $W_{n}:=\pi_{n}: \Omega \rightarrow \Upsilon$ be the canonical projection for any $n \in \mathbb{N}$. Clearly, assertion (iii) of Theorem 4.3 is satisfied by $\left\{W_{n}\right\}_{n \in \mathbb{N}}$, and so, we equivalently get that assertions (iv) and (i) of the same theorem also hold true. Furthermore, it can be easily seen that (iv) $\Longrightarrow$ (vi) as in Theorem 4.3 . hence (iv) $\Longleftrightarrow$ (vi).

Applying similar arguments with those in Example 1, we obtain that assertions (v) and (ii) of Theorem 4.3 are not valid.

The next counterexample shows that the countability assumption for $\Sigma$ in Theorem 4.3 is essential for the equivalence of items (i) to (vi) of Theorem 4.3

Counterexample 2. Let $(\Upsilon, T, Q)$ and $(\Omega, \widetilde{\Sigma}, \widetilde{P})$ be as in Example 1, let $\Sigma$ be the completion of $\widetilde{\Sigma}$ with respect to $\widetilde{P}$ and $P$ the completion of $\widetilde{P}$, and let $\left\{W_{n}\right\}_{n \in \mathbb{N}}$ be as in Example 1 . Then $P$ is a perfect probability measure on $\Sigma$ but $\Sigma$ is not countably generated.

It then follows that assertion (iii) of Theorem 4.3 holds true, while by the same theorem, we get that its assertions (i), (iii) and (iv) are all equivalent. Furthermore, it can be easily shown that (iv) implies (vi). But using similar arguments as in Example 2, we get that assertion (ii) of Theorem 4.3 is not valid.

Assume that assertion (v) of Theorem 4.3 holds true. It then follows that there exist a $\sigma$ subalgebra $\mathcal{F}$ of $\Sigma$ and a subfield $\operatorname{rcp}\left\{S_{\omega}\right\}_{\omega \in \Omega}$ of $P$ over $R:=P \nmid \mathcal{F}$.

There exists a countably generated $\sigma$-subalgebra $\mathcal{A}$ of $\mathcal{F}$ such that $\left\{S_{\omega}\right\}_{\omega \in \Omega}$ satisfies

$$
\int_{F} S_{\omega}(E) R(\mathrm{~d} \omega)=P(E \cap F) \quad \text { for all } F \in \mathcal{A} \text { and } E \in \Sigma \text {. }
$$

and

$$
\forall H \in \Sigma \exists N_{H} \in \mathcal{F} \text { with } P\left(N_{H}\right)=0 \quad S_{\bullet}(H) \uparrow\left(N_{H}\right)^{c} \text { is } \mathcal{A} \text {-measurable. }
$$

In fact, applying similar arguments with those in the proof of Proposition 4.1, we get a countably generated $\sigma$-subalgebra $\widetilde{\mathcal{A}}$ of $\mathcal{F}$ such that for any fixed $\widetilde{H} \in \widetilde{\Sigma}$ the function $S_{\bullet}(H)$ is $\widetilde{\mathcal{A}}$-measurable. Since for $R$-a.a. $\omega \in \Omega$ the measures $P$ and $S_{\omega}$ have the same null sets, we obtain that for any $H \in \Sigma$ there exist sets $\widetilde{H} \in \widetilde{\Sigma}, M_{H} \in \Sigma$ and $N_{H} \in \mathcal{F} \cap \Sigma_{0}$ such that $H=\widetilde{H} \cup M_{H}$ and 
$S_{\omega}\left(M_{H}\right)=P\left(M_{H}\right)=0$ for all $\omega \notin N_{H}$; hence $\left\{S_{\omega}\right\}_{\omega \in \Omega}$ satisfies 5.2 with $\widetilde{\mathcal{A}}$ in the place of $\mathcal{A}$. Applying a monotone class argument, we get a $P$-null set $N_{1} \in \mathcal{F}$ such that for any fixed $\widetilde{H} \in \widetilde{\mathcal{A}}$ the function $S_{\bullet}(\widetilde{H}) \uparrow\left(N_{1}\right)^{c}$ is $\widetilde{\mathcal{A}}$-measurable.

Put $\mathcal{A}:=\sigma\left(\widetilde{\mathcal{A}} \cup\left\{N_{1}\right\}\right) \subseteq \mathcal{F}$. It then follows that $\mathcal{A}$ is countably generated and $\left\{S_{\omega}\right\}_{\omega \in \Omega}$ satisfies (5.1) and (5.2). In particular, for any fixed $H \in \mathcal{A}$ the function $S_{\bullet}(H) \uparrow\left(N_{1}\right)^{c}$ is $\mathcal{A}$-measurable.

Applying now a monotone class argument, we obtain a $P$-null set $N_{2} \in \mathcal{A}$ such that for any $H \in \mathcal{A}$ and $\omega \in N^{c} \cap H$, where $N:=N_{1} \cup N_{2}$, condition $S_{\omega}(H)=1$ holds true.

Choose a set $D \subseteq N^{c}$ such that $D \notin \mathcal{A}$ but $D \in \Sigma$. Following the same reasoning as in Example 2, we deduce that $D \in \mathcal{A}$, which is impossible by the choice of $D$; hence assertion (v) of Theorem 4.3 is not valid.

Throughout what follows, we put $\widetilde{\Omega}:=\mathbb{R}^{\mathbb{N}}, \Omega:=\widetilde{\Omega} \times \mathbb{R}^{d}, \widetilde{\Sigma}:=\mathfrak{B}(\widetilde{\Omega})$ and $\Sigma:=\widetilde{\Sigma} \otimes Z$ for simplicity. The next result extends Theorem 3.1 from [14, which provides a construction for MPPs.

Example 3. Following the reasoning of Theorem 3.1 from 14 but with $\mathbb{R}^{d}, \mathfrak{B}$ and $Q_{n}(\theta)=\mathbf{K}(\theta)$ in the place of $\Upsilon:=(0, \infty), \mathfrak{B}(\Upsilon)$ and $Q_{n}(\theta)=\mathbf{E x p}(\theta)$, respectively, the existence of $(P, \mathbf{K}(\Theta))$ MRPs with prescribed distributions for their interarrival processes as well as for the parameter $\Theta$ is proven.

In fact, fix on arbitrary $\theta \in \mathbb{R}^{d}$ and put $\widetilde{P}_{\theta}:=\otimes_{n \in \mathbb{N}} Q_{n}(\theta)$. Since by assumption, for any fixed $B \in \mathfrak{B}$ each function $Q_{n}(\cdot)(B)$ is $Z$-measurable, it follows by a monotone class argument that the same holds true for the function $\widetilde{P}_{\bullet}(E)$ for fixed $E \in \widetilde{\Sigma}$.

For each $\theta \in \mathbb{R}^{d}$ put $P_{\theta}:=\widetilde{P}_{\theta} \otimes \delta_{\theta}$, where $\delta_{\theta}$ is the Dirac probability measure on $Z$, and for each $n \in \mathbb{N}$ set $W_{n}:=\pi_{n}$, where $\pi_{n}$ is the canonical projection from $\Omega$ onto $\mathbb{R}$. Put now

$$
P(E):=\int \widetilde{P}_{\theta}\left(E^{\theta}\right) \mu(\mathrm{d} \theta) \text { for each } E \in \Sigma,
$$

where $E^{\theta}:=\{\omega \in \Omega:(\omega, \theta) \in E\}$. Then $P$ is a probability measure on $\Sigma$ such that $\left\{P_{\theta}\right\}_{\theta \in \mathbb{R}^{d}}$ is an rcp of $P$ over $\mu$ consistent with $\pi_{\mathbb{R}^{d}}$, where $\pi_{\mathbb{R}^{d}}$ is the canonical projection from $\Omega$ onto $\mathbb{R}^{d}$ (see 14: proof of Theorem 3.1]). Furthermore, it can be proven that for all $\theta \in \mathbb{R}^{d}$ the process $\left\{W_{n}\right\}_{n \in \mathbb{N}}$ is $P_{\theta}$-independent and $\left(P_{\theta}\right)_{W_{n}}=\mathbf{K}(\theta)$ for each $n \in \mathbb{N}$. Clearly, putting $\Theta:=\pi_{\mathbb{R}^{d}}$, we get $P_{\Theta}=\mu$.

It then follows that the counting process $\left\{N_{t}\right\}_{t \in \mathbb{R}_{+}}$induced by $\left\{W_{n}\right\}_{n \in \mathbb{N}}$ (cf. e.g., 21: Theorem 2.1.1]) is a $\left(P_{\theta}, \mathbf{K}(\theta)\right)$-RP for all $\theta \in \mathbb{R}^{d}$; hence by Proposition 3.1 it is a $(P, \mathbf{K}(\Theta))$-MRP.

Applying now Example 3, we compute the probability measures of the corresponding rcp $\left\{P_{\theta}\right\}_{\theta \in \mathbb{R}^{d}}$ as well as the probability measure $P$ for some MRPs of special interest which are not MPPs. To this aim recall that by $\lambda_{d}$ is denoted the restriction of the Lebesgue measure $\lambda_{d}$ to $\mathfrak{B}_{d}$, while any restriction of $\lambda_{d}$ to $\mathfrak{B}(A)$, where $A$ is any Borel subset of $\mathbb{R}^{d}$, will be denoted again by $\lambda_{d}$. In particular, if $d=1$ then $\lambda:=\lambda_{1}=\lambda\lceil\mathfrak{B}$, where $\lambda$ is the Lebesgue measure on $\mathbb{R}$.

In the next example, a concrete $(P, \mathbf{K}(\Theta))$-MRP is constructed for one of the most common choices that can be made for an interarrival time distribution, i.e. $\mathbf{G a}\left(\theta_{1}, \theta_{2}\right)$ with $\theta_{1}>0$ and $\theta_{2}=1 / 2 \in(0,1)$, see e.g. [8 p. 95]. In fact, this class of distributions is of special interest, since none of its members satisfy Assumption 5.1 from 8, proposed by Huang in [11. Theorem 3], which is essential in Grandell's study for MRPs (see [8. Section 5.3]). Moreover, in the same example it is shown that there are counting processes $\left\{N_{t}\right\}_{t \in \mathbb{R}_{+}}$being both $(P, \mathbf{K}(\Theta))$-MRPs and $P_{\Theta}\lceil B(\mathbb{R})$-ones, which are not, though, MRPs according to Grandell 8: Definition 5.3]. 
Example 4. Let $Q_{n}(\theta)=\mathbf{G a}(\theta, 1 / 2)$ for each $n \in \mathbb{N}$ and for any fixed $\theta>0$, and let $\mu=\mathbf{G a}(\gamma, \alpha)$. Then the conclusions of Example 3 are fulfilled for $d=1$; hence $\widetilde{\Omega}=\mathbb{R}^{\mathbb{N}}, \Omega=\mathbb{R}^{\mathbb{N}} \times \mathbb{R}$, while $\widetilde{\Sigma}$, $\Sigma, \widetilde{P}, P,\left\{\widetilde{P}_{\theta}\right\}_{\theta>0},\left\{P_{\theta}\right\}_{\theta>0}$ and $\Theta$ are as in Example 3 .

We first compute the probability measures on measurable cylinders. Let $\widetilde{\mathcal{C}}$ denote the family of all measurable cylinders $\widetilde{B} \in \mathfrak{B}(\widetilde{\Omega})$, i.e. of all sets $\widetilde{B} \subseteq \widetilde{\Omega}$ expressible as $\prod_{n \in \mathbb{N}} \widetilde{B}_{n}$, where $\widetilde{B}_{n} \in \mathfrak{B}$ for every $n \in \mathbb{N}$, and $\widetilde{L}:=\left\{n \in \mathbb{N}: \widetilde{B}_{n} \neq \mathbb{R}\right\}$ is finite. Set $\widetilde{C}_{n}=\widetilde{B}_{n}$ for $n \in \widetilde{L}$. Then $\widetilde{B}=\prod_{k \in \widetilde{L}} \widetilde{C}_{k} \times \mathbb{R}^{\mathbb{N} \backslash \widetilde{L}}$, so we get

$$
\widetilde{P}_{\theta}(\widetilde{B})=\left(\bigotimes_{n \in \mathbb{N}} Q_{n}(\theta)\right)(\widetilde{B})=\prod_{k \in \widetilde{L}} Q_{k}(\theta)\left(\widetilde{C}_{k}\right)=\sqrt{\frac{\theta}{\pi}} \prod_{k \in \widetilde{L}} \int_{\widetilde{C}_{k}} \omega_{k}^{-\frac{1}{2}} \mathrm{e}^{-\theta \omega_{k}} \lambda\left(\mathrm{d} \omega_{k}\right)
$$

for each $\theta>0$. Consider now a measurable cylinder $\widetilde{B} \times E \in \widetilde{\mathcal{C}} \times \mathfrak{B}((0, \infty))$. Applying (5.3), we get

$$
P_{\theta}(\widetilde{B} \times E)=\widetilde{P}_{\theta}(\widetilde{B}) \delta_{\theta}(E)=\chi_{E}(\theta) \sqrt{\frac{\theta}{\pi}} \prod_{k \in \widetilde{L}} \int_{\widetilde{C}_{k}} \omega_{k}^{-\frac{1}{2}} \mathrm{e}^{-\theta \omega_{k}} \lambda\left(\mathrm{d} \omega_{k}\right) ;
$$

for each $\theta>0$. Hence,

$$
P(\widetilde{B} \times E)=\frac{\gamma^{\alpha}}{\Gamma(\alpha) \sqrt{\pi}} \int_{E}\left[\prod_{k \in \widetilde{L} \widetilde{C}_{\widetilde{C}_{k}}} \omega_{k}^{-\frac{1}{2}} \mathrm{e}^{-\theta\left(\gamma+\omega_{k}\right)} \lambda\left(\mathrm{d} \omega_{k}\right)\right] \theta^{\alpha-\frac{1}{2}} \lambda(\mathrm{d} \theta) .
$$

As a consequence, by applying standard methods of Topological Measure Theory, the probability measures $P(E)$ and $P_{\theta}(E)$ can be computed for any $E \in \Sigma$. For details see [14: Example 3.3, (b)].

Finally, it follows an example to show that, we cannot avoid including in Huang's definition of an MRP the assumption that $\left\{W_{n}\right\}_{n \in \mathbb{N}}$ is $P_{\widetilde{y}}$-identically distributed for $\nu$-a.a. $\widetilde{y} \in \widetilde{\Upsilon}$ (see also Remark 44.

Example 5. Let $d=1$. If $Q_{n}(\theta)=\operatorname{Exp}(n \theta)$ for each $n \in \mathbb{N}$ and for any fixed $\theta>0$, and if $\mu=\mathbf{G a}(2,1)$ then all requirements of Example 3 except for

$$
Q_{n}(\theta)=\mathbf{K}(\theta) \text { for all } n \in \mathbb{N} \text { and for any fixed } \theta>0
$$

are satisfied. In fact, in this case $\mathbf{K}(\theta)$ is substituted by $\mathbf{K}(n \theta):=\mathbf{E x p}(n \theta)$.

So the probability measures $\widetilde{P}$ and $P$ on $\widetilde{\Sigma}=\mathfrak{B}(\widetilde{\Omega})$ and $\Sigma=\mathfrak{B}(\Omega)$, where $\widetilde{\Omega}=\mathbb{R}^{\mathbb{N}}$ and $\Omega=\mathbb{R}^{\mathbb{N}} \times \mathbb{R}$, respectively, as well as the rcps $\left\{\widetilde{P}_{\theta}\right\}_{\theta>0}$ and $\left\{P_{\theta}\right\}_{\theta>0}$ can be computed. Moreover, there exists a random variable $\Theta$ on $\Omega$ such that $P_{\Theta}=\mathbf{G a}(2,1)$. Following the same reasoning as in Example 3, we also obtain an interarrival process $\left\{W_{n}\right\}_{n \in \mathbb{N}}$ which is $P_{\theta}$-independent for all $\theta>0$ and satisfies $W_{n}=\pi_{n}$ as well as $\left(P_{\theta}\right)_{W_{n}}=\mathbf{K}(n \theta)$ for all $n \in \mathbb{N}$ and for any fixed $\theta>0$.

But the $P_{\theta}$-independence of $\left\{W_{n}\right\}_{n \in \mathbb{N}}$, for all $\theta>0$, implies for every $r \in \mathbb{N}$ and for all $w_{1}, \ldots, w_{r} \in \mathbb{R}_{+}$that

$$
P\left(\bigcap_{k=1}^{r}\left\{W_{k} \leq w_{k}\right\}\right)=\int \prod_{k=1}^{r} P_{\theta}\left(W_{k} \leq w_{k}\right) \nu(\mathrm{d} \theta)
$$

where $\nu=P_{\Theta}\left\lceil B((0, \infty))=\mu\left\lceil B((0, \infty))\right.\right.$ and $B((0, \infty))=\sigma\left(\left\{P_{\bullet}(E): E \in \Sigma\right\}\right)$. So, $\left\{W_{n}\right\}_{n \in \mathbb{N}}$ is an interarrival process which is not $P_{\theta}$-identically distributed for any fixed $\theta>0$ but which satisfies (5.4). As a consequence, the counting process $\left\{N_{t}\right\}_{t \in \mathbb{R}_{+}}$induced by the sequence of canonical 


\section{SOME CHARACTERIZATIONS OF MRPS}

projections $\left\{\pi_{n}\right\}_{n \in \mathbb{N}}=\left\{W_{n}\right\}_{n \in \mathbb{N}}$ (cf. e.g., 21: Theorem 2.1.1]) is not a $\nu$-MRP associated with $\left\{P_{\theta}\right\}_{\theta>0}$. Furthermore, for every $w_{1}, w_{2} \in \mathbb{R}_{+}$, we have

$$
\begin{aligned}
P\left(W_{1} \leq w_{1}, W_{2} \leq w_{2}\right) & =2 \int_{0}^{\infty}\left(1-\mathrm{e}^{-\theta w_{1}}\right)\left(1-\mathrm{e}^{-2 \theta w_{2}}\right) \mathrm{e}^{-2 \theta} \mathrm{d} \theta \\
& =w_{2}\left(w_{2}+1\right)^{-1}-2\left[\left(w_{1}+2\right)^{-1}-\left(w_{1}+2 w_{2}+2\right)^{-1}\right],
\end{aligned}
$$

implying that $P\left(W_{1} \leq 2, W_{2} \leq 1\right)=\frac{1}{3} \neq \frac{2}{7}=P\left(W_{1} \leq 1, W_{2} \leq 2\right)$; hence $\left\{W_{n}\right\}_{n \in \mathbb{N}}$ is not $P$-exchangeable.

\section{REFERENCES}

[1] COHN, D. L.: Measure Theory. Advanced Texts, 2nd edition, Birkhäuser, 2013.

[2] DELBAEN, F.-HAEZENDONCK, J.: A martingale approach to premium calculation principles in an arbitrage free market, Insurance: Math. Econom. 8 (1989), 269-277.

[3] DIACONIS, P.-FREEDMAN, D.: Finite exchangeable sequences, Ann. Probab. 8 (1980), 745-764.

[4] DUDLEY, R. M.: Real Analysis and Probability, Wadsworth \& Brooks/Cole, Advanced Books \& Software, Pacific Grove, California, 1989.

[5] FADEN, A. M.: The existence of regular conditional probabilities: Necessary and sufficient conditions, Ann. Probab. 13 (1985), 288-298.

[6] FREMLin, D. H.: Measure Theory, Measure Algebras, Vol. 3, Torres Fremlin, Colchester, 2002.

[7] FREMLIN, D. H.: Measure Theory, Topological Measure Spaces, Vol. 4, Torres Fremlin, Colchester, 2003.

[8] GRANDELL, J.: Mixed Poisson Processes, Chapman \& Hall, 1997.

[9] HEWITT, E.-SAVAGE, L. J.: Symmetric measures on Cartesian products, Trans. Amer. Math. Soc. 80 (1955), 470-501.

[10] HOFFmAnn-JØRGEnSEn, J.: The Theory of Analytic Spaces. Various Publications Series, No. 10, Aarhus: Aarhus Universitet, Matematisk, 1970.

[11] HUANG, W. J.: On the characterization of point processes with the exchangeable and Markov properties, Sankhyā A 52 (1990), 16-27.

[12] KAllenBeRG, O.: Probabilistic Symmetries and Invariance Principles, Springer, 2005.

[13] LYBEROPOUlOS, D. P.-MACHERAS, N. D.: Some characterizations of mixed Poisson processes, Sankhyā A 74 (2012), 57-79.

[14] LYBEROPOULOS, D. P.-MACHERAS, N. D.: A construction of mixed Poisson processes via disintegrations, Math. Slovaca 63(1) (2013), 167-182.

[15] LYBEROPOULOS, D. P.-MACHERAS, N. D.: A characterization of martingale-equivalent mixed compound Poisson processes, Ann. Appl. Probab. 31(2) (2021), 778-805.

[16] LYBEROPOUlOS, D. P.-MACHERAS, N. D.-TZANINIS, S. M.: On the equivalence of various definitions of mixed Poisson processes, Math. Slovaca 69(2) (2019), 453-468.

[17] MACHERAS, N. D.-TZANINIS, S. M.: Some characterizations for Markov processes as mixed renewal processes, Math. Slovaca 68(6) (2018), 1477-1494.

[18] OLSHEN, R.: A note on exchangeable sequences, Z. Wahrscheinlichkeitstheorie verw. Geb. 28 (1974), $317-321$.

[19] PACHL, J. K.: Disintegration and compact measures, Math. Scand. 43 (1978), 157-168.

[20] PROTTER, PH. E.: Stochastic Integration and Differential Equations, 2nd ed., Springer, 2004.

[21] SCHMIDT, K. D.: Lectures on Risk Theory, B. G. Teubner, Stuttgart, 1996.

[22] SEGERDAHL, C.-O.: Stochastic processes and practical working models or why is the Pólya process approach defective in modern practice and how to cope with its deficiencies? Scand. Actuar. J. 1970 (1970), 146-166.

[23] STRAUSS, W.-MACHERAS, N. D.-MUSIAL, K.: Splitting of liftings in products of probability spaces, Ann. Probab. 32 (2004), 2389-2408.

[24] TZANINIS, S. M.-MACHERAS, N. D.: A characterization of equivalent martingale probability measures in a mixed renewal risk model with applications in Risk Theory, https://arxiv.org/abs/2007.09051 
DEMETRIOS P. LYBEROPOULOS - NIKOLAOS D. MACHERAS

Received 5. 6. 2020

Accepted 11. 1. 2021

\author{
*Hellenic Statistical Authority (ELSTAT) \\ 46 Pireos str. Eponiton str. \\ 18510 Piraeus \\ GREECE \\ E-mail: d.lymperopoulos@statistics.gr \\ dilyber@webmail.unipi.gr \\ ** Department of Statistics and Insurance Science \\ University of Piraeus \\ 80 Karaoli and Dimitriou str. \\ 18534 Piraeus \\ GREECE \\ E-mail: macheras@unipi.gr
}

